\title{
Gravitational and gauge couplings in Chern-Simons fractional spin gravity
}

\author{
Nicolas Boulanger, ${ }^{a, b, 1}$ Per Sundell ${ }^{c}$ and Mauricio Valenzuela ${ }^{d}$ \\ ${ }^{a}$ Mécanique et Gravitation, Université de Mons - UMONS, \\ 20 Place du Parc, 7000 Mons, Belgique \\ ${ }^{b}$ Laboratoire de Mathématiques et Physique Théorique, Unité Mixte de Recherche 7350 du CNRS, \\ Université François Rabelais, \\ Parc de Grandmont, 37200 Tours, France \\ ${ }^{c}$ Departamento de Ciencias Físicas, Universidad Andres Bello, \\ Republica 220, Santiago, Chile \\ ${ }^{d}$ Facultad de Ingeniería y Tecnología, Universidad San Sebastían, \\ General Lagos 1163, Valdivia 5110693, Chile \\ E-mail: nicolas.boulanger@umons.ac.be, per.sundell@unab.cl, \\ valenzuela.u@gmail.com
}

ABSTRACT: We propose an extension of Vasiliev's supertrace operation for the enveloping algebra of Wigner's deformed oscillator algebra to the fractional spin algebra given in arXiv:1312.5700. We provide a necessary and sufficient condition for the consistency of the supertrace, through the existence of a certain ground state projector. We build this projector and check its properties to the first two orders in the number operator and to all orders in the deformation parameter. We then find the relation between the gravitational and internal gauge couplings in the resulting unified three-dimensional ChernSimons theory for Blencowe-Vasiliev higher spin gravity coupled to fractional spin fields and internal gauge potentials. We also examine the model for integer or half-integer fractional spins, where infinite dimensional ideals arise and decouple, leaving finite dimensional gauge algebras $g l(2 \ell+1)$ or $g l(\ell \mid \ell+1)$ and various real forms thereof.

Keywords: Higher Spin Symmetry, Chern-Simons Theories, Anyons

ArXiv EPrint: 1504.04286

\footnotetext{
${ }^{1}$ Associate researcher of the FNRS, Belgium.
} 


\section{Contents}

1 Introduction $\quad 1$

1.1 General background 1

1.2 Problem setting and main results 2

2 Definition of the model $\quad 4$

2.1 Deformed oscillator enveloping algebra and supertrace 4

$\begin{array}{lll}2.2 & \text { Non-polynomial extension and fractional spin algebra } & 6\end{array}$

2.3 Discrete generators, trace operation and Chern-Simons action $\quad 7$

3 Construction of quasi-projectors $\quad 9$

$\begin{array}{ll}3.1 & \text { Creation and annihilation operator basis } \\ \end{array}$

3.2 Non-critical versus critical $\nu \quad 11$

$\begin{array}{lll}3.3 & \text { Projectors in non-critical case } & 11\end{array}$

3.4 Quasi-projectors for critical $\nu= \pm 3, \pm 5, \ldots \quad 15$

3.5 Example: $\nu=-3 \quad 18$

3.6 The hypercritical cases: $\nu= \pm 1 \quad 20$

4 Final specification of the model $\quad 21$

4.1 Real forms 21

$4.2 \tau$ projections $\quad 22$

4.3 Final form of action and couplings 23

5 Conclusion $\quad 24$

$\begin{array}{ll}\text { A Conventions } & 25\end{array}$

\section{Introduction}

\subsection{General background}

Higher spin gravity $[1,2]$ was originally aimed as a theory generalizing gravitational interactions to arbitrary spin, understood to be integer or half-an-integer. However, in constantly curved $2+1$-dimensional backgrounds, one may lift this restriction, as there the isometry algebras admit physical representations with fractional spins $[3,4]$, i.e. spins interpolating between half-integer and integer numbers, which can be carried by particles carrying anyon statistics $[5,6]$; see [7] for a review. Thus, aiming at a complete description of interacting relativistic fields in three dimensions, it is natural to ask whether fractional spin fields can be coupled to the gravitational field and internal gauge fields. 
In [8], we proposed an action describing non-Abelian interactions among fractional spin fields, tensorial higher spin fields and internal gauge fields using a flat connection valued in a fractional spin algebra. The tensorial higher spin gauge fields are packaged into a Blencowe-Vasiliev $[9,10]$ master one-form $W$ valued in a bosonic higher spin algebra forming a subalgebra of the fractional spin algebra and whose basis is given in terms of monomials in Wigner-deformed oscillators. Likewise, the internal gauge fields make up a bosonic one-form $U$ valued in a compact real form of an infinite dimensional matrix algebra, formally isomorphic to an algebra of Fock space endomorphisms. As for the fractional spin fields, they are collected into two master fields $(\psi, \bar{\psi})$ that intertwine the gravitational and internal gauge algebras. Thus, the model constructed in [8] can be regarded in two ways: either as an extension of the Blencowe-Vasiliev theory $[9,10]$ by internal and fractional spin fields, or as a formal (real) analytical continuation of Chern-Simons supergravity in $2+1$ dimensions $[11,12]$ whereby the gravitino fields are extended into fractional spin fields, thus forcing the introduction of fields with spin greater than two.

On shell, the consistency of the construction relies on the associativity of the underlying fractional spin algebra, which was demonstrated in [8] using Fock space methods. Off shell, however, the action requires a trace operation, whose precise form was conjectured in [8], and whose explicit construction we shall tend to below. Our construction is facilitated by extending Vasiliev's supertrace operation [10] on the higher spin algebra from its original domain of validity, which is the space of polynomials in the generators of the deformed oscillator algebra, to the space of non-polynomial elements that spans the internal gauge algebra and the intertwiners. As a concrete application of this result, we compute the explicit relation between the gravitational and gauge couplings in the resulting unified model.

\subsection{Problem setting and main results}

The field content of the model can be assembled into a matrix master field

$$
\mathbb{A}=\left[\begin{array}{ll}
W & \psi \\
\bar{\psi} & U
\end{array}\right],
$$

valued in an associative ${ }^{1}$ fractional spin algebra $\mathcal{A}_{ \pm}$, containing semiclassical bosonic $(-)$ or fermionic $(+)$ fractional spin fields. The action proposed in [8] is of the standard ChernSimons format, viz.

$$
S_{ \pm}[\mathbb{A}]=\frac{\varkappa}{2 \pi} \int_{M_{3}} \operatorname{Tr}_{\mathcal{A}_{ \pm}}\left(\frac{1}{2} \mathbb{A} \star d \mathbb{A}+\frac{1}{3} \mathbb{A} \star \mathbb{A} \star \mathbb{A}\right)
$$

where thus the key ingredients are the associative product $\star$ (including the wedge product) and a non-degenerate cyclic trace operation $\operatorname{Tr}_{\mathcal{A}_{ \pm}}$. Assuming their salient features, the resulting equations of motion read

$$
d \mathbb{A}+\mathbb{A} \star \mathbb{A}=0,
$$

\footnotetext{
${ }^{1}$ The model can be projected further to a model based on a Lie algebra. However, thinking of it as a truncation of a larger theory containing also matter fields, the notion of an associative gauge algebra becomes crucial.
} 
or in components,

$$
\begin{aligned}
& d W+W \star W+\psi \star \bar{\psi}=0, \quad d U+U \star U+\bar{\psi} \star \psi=0, \\
& d \psi+W \star \psi+\psi \star U=0, \quad d \bar{\psi}+U \star \bar{\psi}+\bar{\psi} \star W=0 .
\end{aligned}
$$

As found in [8], the standard bra-ket formalism for Fock space endomorphisms can be augmented by a set of fusion rules, reflecting the couplings in eqs. (1.4)-(1.5), that suffice for on-shell consistency. More precisely, the fusion rules stipulate how to perform the star products in eqs. (1.4) and (1.5) and expand the results in the bases stipulated by the linear terms while preserving associativity so as to achieve a Cartan integrable system.

The off-shell formulation, however, requires an algebraic machinery that facilitates a trace operation that applies to both higher spin and internal matrix subalgebras. The standard (unregularized) Fock space trace operation does not suffice as it does not apply straightforwardly to polynomials in deformed oscillators. ${ }^{2}$ Instead, in [8], it was proposed to realize the internal matrix subalgebra of $\mathcal{A}_{ \pm}$using real-analytic non-polynomial symbols and obtaining $\operatorname{Tr}_{\mathcal{A}_{ \pm}}$by extending Vasiliev's supertrace operation [10] correspondingly, beyond its original domain of validity (given by the algebra of arbitrary polynomials in the deformed oscillators). To this end, in order to demonstrate the salient features of the star product and trace operation on $\mathcal{A}_{ \pm}$, it suffices to establish the following conditions:

i) Finite star products in $\mathcal{A}_{ \pm}$(from which associativity follows);

ii) Finite Vasiliev supertraces (which together with (i) implies cyclicity).

In what follows, we shall show that the matrix subalgebra of $\mathcal{A}_{ \pm}$is realized in terms of confluent hypergeometric functions of the spin operator, which establishes (ii). We shall also verify a necessary condition for (i) to hold true, namely that the supertrace of the square of two ground state projectors is finite, hoping to present a complete proof together with a construction of a convolution formula for the deformed oscillator star product in Weyl order and a related trace formula in a future work.

Moreover, assuming the consistency of the model, and focusing on the fermion model (with internal $k$-parity $\sigma=-1$ ), we shall compute the aforementioned relations between couplings, viz.

$$
k_{\mathrm{hs}}=\frac{\varkappa}{16}\left(1-\nu^{2}\right)\left(1-\frac{\nu}{3}\right), \quad k_{\mathrm{int}}=\varkappa,
$$

between the fractional spin coupling $\varkappa$ defined in (1.2), the higher spin coupling $k_{\mathrm{hs}}$ (related to Newton's constant $G_{N}$ as in (4.24)) and the level $k_{\text {int }}$ of the internal gauge theory.

The paper is organised as follows: in section 2, we define the algebraic structures of our model. In section 3, we construct the symbols of the basis elements of the internal and fractional spin sectors of the theory, analyse the properties of the extension of Vasiliev's trace operation and consider critical limits. Reality conditions and various projections are studied in section 4 , where we also establish the aforementioned relation between the gravitational and internal couplings. Finally, we conclude in section 5. Our three-dimensional spinor conventions are given in the appendix.

\footnotetext{
${ }^{2}$ To our best understanding, it remains unclear whether a regularized Fock space trace operation could be used to construct $\operatorname{Tr}_{\mathcal{A}_{ \pm}}$.
} 


\section{Definition of the model}

In this section we detail the basic algebraic structures going into the model.

\subsection{Deformed oscillator enveloping algebra and supertrace}

The higher spin fields are thus collected in a Blencowe-Vasiliev master one-form

$$
W \in \mathcal{W}^{++} \otimes \operatorname{Cliff}(\gamma)
$$

where $\gamma$ is an idempotent element introduced to account for the anti-de Sitter translations, and $\mathcal{W}^{++}$is an associative algebra given by a certain non-polynomial extension, to be spelled out in section 2.2, of the enveloping algebra $A q(2 ; \nu)[10]$ of the deformed oscillator algebra $[13,14]^{3}$ (see also $\left.[15,16]\right)$

$$
\begin{aligned}
& {\left[q_{\alpha}, q_{\beta}\right]_{\star}=2 i(1+\nu k) \epsilon_{\alpha \beta}, \quad\left\{k, q_{\alpha}\right\}_{\star}=0, \quad k \star k=1,} \\
& \left(q_{\alpha}\right)^{\dagger}=q_{\alpha}, \quad k^{\dagger}=k, \quad \nu \in \mathbb{R} .
\end{aligned}
$$

By its definition, the algebra $A q(2 ; \nu)$ consists of arbitrary star polynomials (of finite degree) in $\left(q_{\alpha}, k\right)$. In addition to the hermitian conjugation, which acts as $(f \star g)^{\dagger}=g^{\dagger} \star f^{\dagger}$, this algebra has a linear anti-involution $\tau$ defined by

$$
\tau(f \star g)=\tau(g) \star \tau(f), \quad \tau\left(q_{\alpha}, k\right)=\left(i q_{\alpha}, k\right) .
$$

In general, certain algebraic properties of infinite-dimensional associative algebras, such as unitarity and indecomposability of representations, crucially depend on the choice of basis. As basis for $A q(2 ; \nu)$ we choose the Weyl-ordered elements

$$
T_{\alpha(n)}:=q_{\alpha_{1}} \cdots q_{\alpha_{n}} \equiv q_{\left(\alpha_{1}\right.} \star \cdots \star q_{\left.\alpha_{n}\right)},
$$

and $T_{\alpha(n)} \star k$, where the symmetrization has unit strength. Equivalently, one may use the projected elements

$$
T_{\alpha(n)}^{\sigma, \sigma^{\prime}}:=\left[q_{\alpha_{1}} \cdots q_{\alpha_{n}}\right]^{\sigma, \sigma^{\prime}}:=\Pi^{\sigma} \star q_{\left(\alpha_{1}\right.} \star \cdots \star q_{\left.\alpha_{n}\right)} \star \Pi^{\sigma^{\prime}}, \quad \Pi^{ \pm}=\frac{1}{2}(1 \pm k),
$$

which are non-vanishing iff $\sigma \sigma^{\prime}=(-1)^{n}$. Correspondingly, we define the projections

$$
A q(2 ; \nu)^{\sigma, \sigma^{\prime}}=\Pi^{\sigma} \star A q(2 ; \nu) \star \Pi^{\sigma^{\prime}} .
$$

One may thus represent the elements $f \in A q(2 ; \nu)$ by polynomials in the semi-classical basis elements $q_{\alpha_{1}} \cdots q_{\alpha_{n}}$ and $q_{\alpha_{1}} \cdots q_{\alpha_{n}} k:=q_{\alpha_{1}} \cdots q_{\alpha_{n}} \star k$, referred to as their Weyl ordered symbols, and which we shall denote by $f$ as well, in a slight abuse of the otherwise more involved notation. Thus, using this representation, the operator product amounts to a

\footnotetext{
${ }^{3}$ As our analysis only relies on the fundamental relations given in (2.2), it remains valid for any realization of the deformed oscillators.
} 
non-local composition rule for symbols, which we shall denote by a $\star$ as well. As far as star product compositions of monomials are concerned, they can be deduced by iterating

$$
q_{\alpha} \star T_{\beta(n)}=T_{\alpha \beta(n)}+i n \epsilon_{\alpha\left(\beta_{1}\right.}\left(1+\frac{n+\frac{1}{2}\left(1-(-1)^{n}\right)}{n(n+1)} \nu k\right) \star T_{\beta(n-1))},
$$

or its projected form

$$
q_{\alpha} \star T_{\beta(n)}^{\sigma, \sigma^{\prime}}=T_{\alpha \beta(n)}^{-\sigma, \sigma^{\prime}}+i n \epsilon_{\alpha\left(\beta_{1}\right.}\left(1-\frac{n+\frac{1}{2}\left(1-(-1)^{n}\right)}{n(n+1)} \nu \sigma\right) \star T_{\beta(n-1))}^{-\sigma, \sigma^{\prime}} .
$$

It follows that $A q(2 ; \nu)$ does not contain any ideal for

$$
\text { Non-critical } \nu \notin 2 \mathbb{Z}+1 \text {, }
$$

while for $(\ell=0,1,2, \ldots ; \hat{\sigma}= \pm 1)$

$$
\text { Critical } \nu \equiv(2 \ell+1) \hat{\sigma} \in 2 \mathbb{Z}+1
$$

it contains the ideal

$$
A q^{\prime}(2 ; \nu)=\biguplus_{n \geqslant 0}\left[T_{\alpha(2 \ell+2 n)}^{\hat{\sigma}, \hat{\sigma}} \oplus T_{\alpha(2 \ell+1+2 n)}^{\hat{\sigma},-\hat{\sigma}}\right]
$$

giving rise to the finite dimensional coset

$$
\frac{A q(2 ; \nu)}{A q^{\prime}(2 ; \nu)} \cong g l(2 \ell+1)=g l(\ell)^{\hat{\sigma}, \hat{\sigma}} \oplus g l(\ell+1)^{-\hat{\sigma},-\hat{\sigma}} \Subset\left[(\ell, \ell+1)^{\hat{\sigma},-\hat{\sigma}} \oplus(\ell+1, \ell)^{-\hat{\sigma}, \hat{\sigma}}\right],
$$

where $(\ell, \ell+1)$ and $(\ell+1, \ell)$ denote the $\ell(\ell+1)$-dimensional bi-fundamental representations of $g l(\ell) \oplus g l(\ell+1)$, realized as suitably $\Pi^{ \pm}$-projected odd polynomials.

Turning to Vasiliev's cyclic trace operation on $A q(2 ; \nu)$, it is given by [10]

$$
\operatorname{Tr}_{A q(2 ; \nu)}(\cdot)=\operatorname{STr}_{A q(2 ; \nu)}(k \star(\cdot)),
$$

where the graded cyclic supertrace operation $\operatorname{STr}_{A q(2 ; \nu)}$ is fixed uniquely by its defining properties

$$
\mathrm{STr}_{A q(2 ; \nu)}(f \star g)=\mathrm{STr}_{A q(2 ; \nu)}(g \star k \star f \star k), \quad \operatorname{STr}_{A q(2 ; \nu)}(1)=1 .
$$

Thus, if $f$ has a definite parity, viz. $k \star f \star k=(-1)^{f} f$, then $\operatorname{STr}_{A q(2 ; \nu)}(f \star g)=$ $(-1)^{f} \operatorname{STr}_{A q(2 ; \nu)}(g \star f)$. In the Weyl ordered basis, one has

$$
\mathrm{STr}_{A q(2 ; \nu)} T_{\alpha(n)}^{\sigma, \sigma^{\prime}}=\delta_{n, 0} \delta^{\sigma, \sigma^{\prime}} \frac{(1-\sigma \nu)}{2} .
$$

More compactly, by representing $f$ using its Weyl ordered symbol $f(q, k)$, one has

$$
\operatorname{STr}_{A q(2 ; \nu)}(f)=f(0 ;-\nu),
$$

that is, the trace operation maps the Kleinian $k$ to $-\nu$ inside the symbol. In critical limits, one has

$$
\mathrm{STr}_{A q(2 ; \nu)} A q^{\prime}(2 ; \nu)=0,
$$

which means that in critical limits the model is truncated to

$$
W \in g l\left(\ell+\frac{1}{2}(1+\hat{\sigma})\right) \otimes \operatorname{Cliff}(\gamma) .
$$




\subsection{Non-polynomial extension and fractional spin algebra}

In order to describe the fractional spin model, we extend the enveloping algebra $A q(2 ; \nu)$ into an associative algebra module $M q(2 ; \nu)$ that contains two dual subspaces as follows: We first introduce the formal associative extension $\overline{A q}(2 ; \nu)$ of $A q(2 ; \nu)$ consisting of elements $f$ with Weyl ordered symbols given by power series

$$
f(q, k)=\sum_{m=0}^{\infty} \sum_{n=0,1} f^{\alpha(m)} q_{\alpha_{a}} \cdots q_{\alpha_{n}} k^{n},
$$

that we shall assume are traceable using the natural extension of (2.14). In order to specify this extension, we need to introduce suitable dual basis elements, cf. the addition of points at infinity to a non-compact manifold. The dual elements form a dual associative algebra $A w(2 ; \nu)$, which will turn out to consist of Wigner distributions that have fixed eigenvalues under the one-sided action of the spin operator belonging to lowest or highest weight spaces.

To this end, we choose the Lorentz connection to be the gauge field in $W$ associated with the $s o(1,2) \cong s l(2 ; \mathbb{R})$ algebra generated by

$$
J_{a}=\frac{1}{4}\left(\tau_{a}\right)^{\alpha \beta} J_{\alpha \beta}, \quad J_{\alpha \beta}=\frac{1}{2} q_{(\alpha} \star q_{\beta} \star \Pi^{+},
$$

using the conventions given in appendix A. By this embedding of the Lorentz algebra into the gauge algebra, it follows that $T_{\alpha(n)}^{++}(n=0,2,4, \ldots)$, and hence the corresponding gauge fields, transform in the adjoint representation of spin $n / 2$, which are thus integers. The fields $(\psi, \bar{\psi})$, on the other hand, transform under Lorentz transformations in representations induced by the separate left and right star multiplication by $J_{\alpha \beta}$, respectively. Indeed, by examining the Casimir operator $C_{2} \equiv j(1-j)$, one finds that these representations are characterized by a spin $j=\frac{1+\nu}{4}$, which contains a fractional part, not given by an integer or half-an-integer, except for

$$
\text { critical } \nu \in 2 \mathbb{Z}+1 \text {. }
$$

To specify these representations, we assume that they are equipped by basis states that diagonalize the spatial spin generator ${ }^{4}$

$$
J_{0}=\frac{1}{2} w \star \Pi^{+}, \quad w=\frac{1}{4}\left(\tau_{0}\right)^{\alpha \beta} q_{\alpha} \star q_{\beta} .
$$

Furthermore, for a complete specification, one needs to specify whether and how this operator is bounded or not. For definiteness, we shall take

$$
A w(2 ; \nu)=\bigoplus_{\sigma, \sigma^{\prime} ; \epsilon ; \lambda, \lambda^{\prime}} T_{\lambda|\epsilon| \lambda^{\prime}}^{\sigma, \sigma^{\prime}}
$$

to consist of finite linear combinations of generalized quasi-projectors $T_{\lambda|\epsilon| \lambda^{\prime}}^{\sigma, \sigma^{\prime}} \in \overline{A q}(2 ; \nu)$ obeying the " $\star$-genvalue" equation

$$
(w-\lambda) \star T_{\lambda|\epsilon| \lambda^{\prime}}^{\sigma, \sigma^{\prime}}=0=T_{\lambda|\epsilon| \lambda^{\prime}}^{\sigma, \sigma^{\prime}} \star\left(w-\lambda^{\prime}\right), \quad\left(T_{\lambda|\epsilon| \lambda^{\prime}}^{\sigma, \sigma^{\prime}}\right)^{\dagger}=T_{\lambda^{\prime}|\epsilon| \lambda}^{\sigma^{\prime}, \sigma}, \quad \epsilon= \pm 1,
$$

\footnotetext{
${ }^{4}$ Other classes of fractional spin models arise if one instead chooses to diagonalize a boost or a light-like spin generator.
} 
and belonging to one-sided representations of $A q(2 ; \nu)$ in which $\epsilon w$ is bounded from below. ${ }^{5}$ Thus, accounting for the internal gauge fields as well, one is led to the basic fractional spin algebra $^{6}$

$$
\mathcal{A}(2 ; \nu \mid w):=\left[\begin{array}{cc}
\mathcal{W}^{++} & \mathcal{I}^{+-} \\
\overline{\mathcal{I}}^{-+} & \mathcal{U}^{--}
\end{array}\right],
$$

consisting of the spaces

$$
\begin{aligned}
\mathcal{W}^{++} & =\Pi^{+} \star \overline{A q}(2 ; \nu) \star \Pi^{+}, & \mathcal{U}^{--} & =\Pi^{-} \star \overline{A w}(2 ; \nu) \star \Pi^{-}, \\
\mathcal{I}^{+-} & =\Pi^{+} \star \overline{A w}(2 ; \nu) \star \Pi^{-}, & \overline{\mathcal{I}}^{-+} & =\Pi^{-} \star \overline{A w}(2 ; \nu) \star \Pi^{+},
\end{aligned}
$$

where $\overline{A w}(2 ; \nu)$ is the extension of of $A w(2 ; \nu)$ by infinite-dimensional traceable matrices. The associative product law of $\mathcal{A}(2 ; \nu \mid w)$ is defined by a fusion rule, which one may think of as a germ of an underlying topological open string, that stipulates that: i) the product of an arbitrary polynomial and a quasi-projector is always to be expanded in the basis of quasi-projectors; and ii) the product of two quasi-projectors is to be expanded in terms of the basis of quasi-projectors or the basis of Weyl-ordered monomials in accordance with the sector to which the product belongs. ${ }^{7}$ Thus, returning to the abstract module, we define it formally as

$$
M q(2 ; \nu)=A q(2 ; \nu) \cup(\overline{A q}(2 ; \nu) \cap \overline{A w}(2 ; \nu)),
$$

which thus contains $A q(2 ; \nu)$ and $A w(2 ; \nu)$ as two dual subalgebras. There is an asymmmetry between these two spaces, as the construction overlap requires $A w(2 ; \nu)$ to be mapped to $\overline{A q}(2 ; \nu)$ while it does not require any converse map. In other words, the module, thought of as a manifold, is glued together via a monomorphism $\rho: A w(2 ; \nu) \rightarrow \overline{A q}(2 ; \nu)$. Thus, in order to define the trace operation on $M q(2 ; \nu)$, it suffices to introduce a trace operation $\operatorname{Tr}_{A q(2 ; \nu)}$ on $A q(2 ; \nu)$ and show that it extends to $\rho(A w(2 ; \nu)), v i z . \operatorname{Tr}_{A w(2 ; \nu)}(f)=$ $\operatorname{Tr}_{\overline{A q}(2 ; \nu)}(\rho(f))$, to which we shall turn next.

\subsection{Discrete generators, trace operation and Chern-Simons action}

To treat the cases of Grassmann even or odd fractional spin fields uniformally and to account for anti-de Sitter translations, we introduce a fermionic generator $\xi$ and a bosonic generator $\gamma$ whose non-trivial relations are

$$
\xi \star \xi=1, \quad \gamma \star \gamma=1,
$$

and extend the fractional spin algebra (2.26) into

$$
\mathcal{A}_{ \pm}=[\mathcal{A}(2 ; \nu \mid w) \otimes \operatorname{Cliff}(\gamma) \otimes \operatorname{Cliff}(\xi)]_{ \pm},
$$

\footnotetext{
${ }^{5}$ The algebra $A w(2 ; \nu)$ is a subalgebra of the algebra $A w_{\text {ext }}(2 ; \nu)$ spanned by quasi-projectors $T_{\lambda\left|\left(\epsilon, \epsilon^{\prime}\right)\right| \lambda^{\prime}}^{\sigma, \sigma^{\prime}}$ belonging to one-sided representations of $A q(2 ; \nu)$ in which the left action of $\epsilon w$ and the right action of $\epsilon^{\prime} w$ are bounded from below; the space $A w_{\text {ext }}(2 ; \nu) \backslash A w(2 ; \nu)$ thus consists of quasi-projectors that connect states in lowest (highest) weight spaces to highest (lowest) weight spaces. For example, for $\nu=0$ one has $T_{\frac{\epsilon}{2}|(\epsilon,-\epsilon)|-\frac{\epsilon}{2}}^{\sigma, \sigma}=\pi \delta\left(a^{-\epsilon}\right)$.

${ }^{6}$ In [8] we used an auxiliary Fock space $\mathcal{F}$ to define the fractional spin subalgebra $\mathcal{A}\left(2 ; \nu \mid \mathfrak{o}(2)_{J_{0}} ; \mathcal{F}\right)$ of $A w(2 ; \nu)$ obtained by restricting to the subspace in which $w$ is bounded from below.

${ }^{7}$ The fusion rule does not require that a monomial admits any expansion in the basis of quasi-projectors.
} 
consisting of Grassmann even elements

$$
\mathbb{X}=\left[\begin{array}{ll}
X^{++} & X^{+-} \\
\bar{X}^{-+} & X^{--}
\end{array}\right]
$$

that obey the internal parity condition

$$
\pi_{q} \pi_{\xi}(\mathbb{X})=\mathbb{C}_{ \pm} \star \mathbb{X} \star \mathbb{C}_{ \pm}, \quad \mathbb{C}_{ \pm}=\left[\begin{array}{cc}
1 & 0 \\
0 & \pm 1
\end{array}\right]
$$

where $\pi_{q}$ and $\pi_{\xi}$ are the automorpisms of the star product algebra that reverse the sign of $q_{\alpha}$ and $\xi$, respectively. Thus, the elements $X^{++}$and $X^{--}$are $\xi$ independent and hence $W$ and $U$ have expansions in terms of bosonic component fields. In the case of $\mathcal{A}_{-}$, the same holds for $\left(X^{+-}, \bar{X}^{-+}\right)$and $(\psi, \bar{\psi})$. In the case of $\mathcal{A}_{+}$, the elements $\left(X^{+-}, \bar{X}^{-+}\right)$are linear in $\xi$ and hence $(\psi, \bar{\psi})$ have expansions in terms of fermionic component fields. In other words, the semi-classical statistics of the component fields is correlated with the internal parity defined by the $\pi_{q}$ map, such that the components of parity even elements are bosonic while those of parity odd elements are fermionic in $\mathcal{A}_{+}$and bosonic in $\mathcal{A}_{-}$. More explicitly,

$$
\begin{aligned}
& \text { Fermionic fractional spin fields }\left(\mathcal{A}_{+}\right):(\psi, \bar{\psi})=(\Theta \star \xi, \xi \star \bar{\Theta}), \\
& \text { Bosonic fractional spin fields }\left(\mathcal{A}_{-}\right):(\psi, \bar{\psi})=(\Sigma, \bar{\Sigma}),
\end{aligned}
$$

where thus $\Theta$ and $\Sigma$ have expansions in terms of bosonic symbols in $\overline{A w}^{+,-}(2 ; \nu)$ multiplied by component fields that are fermions and bosons, respectively.

Turning to the trace operation, we use the fact that the fermionic Clifford algebra Cliff $(\xi)$, which by its definition consists of Grassmann even elements of the form $X=$ $X_{0}+X_{1} \xi$, where thus $X_{0}$ is a boson and $X_{1}$ is a fermion, has the supertrace operation ${ }^{8}$ $\operatorname{STr}_{\text {Cliff }(\xi)}(X)=X_{0}$, which thus obeys $\operatorname{STr}_{\operatorname{Cliff}(\xi)}\left(X \star X^{\prime}\right)=\operatorname{STr}_{\operatorname{Cliff}(\xi)}\left(X^{\prime} \star \xi \star X \star \xi\right)$. The bosonic Clifford algebra $\operatorname{Cliff}(\gamma)$, which by its definition consists of Grassmann even elements of the form $Y=Y_{0}+Y_{1} \gamma$, has a two-parameter family of trace operations, namely $\operatorname{Tr}_{\mathrm{Cliff}(\gamma)}^{(y, \tilde{y})}(Y)=\frac{y}{2}\left(Y_{0}+Y_{1}\right)+\frac{\tilde{y}}{2}\left(Y_{0}-Y_{1}\right)$, where $y, \tilde{y} \in \mathbb{R}$.

As for $\overline{A q}(2 ; \nu)$ and $\overline{A w}(2 ; \nu)$, we extend Vasiliev's supertrace operation $\operatorname{STr}_{A q(2 ; \nu)}$ given in (2.17), which is valid for arbitrary polynomials stricto sensu, by a procedure that is formally reminiscent of the fusion rule: the operator in the argument of the trace is first expanded in the Weyl ordered basis (2.20) and the resulting symbol is then evaluated using (2.17). As we shall show below, this extension of $\operatorname{STr}_{A q(2 ; \nu)}$, that we shall denote by $\mathrm{STr}_{\overline{A q}(2 ; \nu)}$, preserves its salient features, viz.

$$
\mathrm{STr}_{\overline{A q}(2 ; \nu)}(f \star g)=\mathrm{STr}_{\overline{A q}(2 ; \nu)}(g \star k \star f \star k),
$$

and if $\operatorname{STr}_{\overline{A q}(2 ; \nu)}(f \star g)=0$ for all $g$ then $f=0$.

\footnotetext{
${ }^{8}$ The supertrace operation, which is intrisically bosonic, induces an intrinsically fermionic trace operation $\operatorname{Tr}_{\mathrm{Cliff}(\xi)}(X)=\mathrm{STr}_{\mathrm{Cliff}(\xi)}(\xi \star X)=-X_{1}$, which does not play any role in the present class of models.
} 
Combining the operations introduced so far with the standard trace operation on $\mathrm{Mat}_{2}$, we are led to equip the extended fractional spin algebra with the following trace operation:

$$
\begin{aligned}
\operatorname{Tr}_{\mathcal{A}_{ \pm}}^{(x)} \mathbb{X} & =\operatorname{Tr}_{\overline{A q}(2 ; \nu)} \operatorname{Tr}_{\operatorname{Cliff}_{1}(\gamma)}^{(1+x,-1+x)} \operatorname{STr}_{\mathrm{Cliff}_{1}(\xi)} \operatorname{Tr}_{\mathrm{Mat}_{2}} \mathbb{X} \star \mathbb{C}_{\mp} \\
& =\operatorname{Tr}_{\overline{A q}(2 ; \nu)} \operatorname{Tr}_{\mathrm{Cliff}_{1}(\gamma)}^{(1+x,-1+x)} \operatorname{STr}_{\mathrm{Cliff}_{1}(\xi)}\left(X^{++} \mp X^{--}\right) \\
& =\left.\operatorname{STr}_{\overline{A q}(2 ; \nu)}\left(k \star \gamma \star\left(X^{++} \mp X^{--}\right)\right)\right|_{\gamma=x} \\
& =\left.\operatorname{STr}_{\overline{A q}(2 ; \nu)}\left(\gamma \star\left(X^{++} \pm X^{--}\right)\right)\right|_{\gamma=x}
\end{aligned}
$$

where $x \in \mathbb{R}$ is a chiral symmetry breaking parameter. In view of the claimed properties of $\operatorname{STr}_{\overline{A q}(2 ; \nu)}$, we thus have

$$
\operatorname{Tr}_{\mathcal{A}_{ \pm}}^{(x)} \mathbb{X} \star \mathbb{X}^{\prime}=\operatorname{Tr}_{\mathcal{A}_{ \pm}}^{(x)} \mathbb{X}^{\prime} \star \mathbb{X}
$$

and that if $\operatorname{Tr}_{\mathcal{A}_{ \pm}}^{(x)} \mathbb{X} \star \mathbb{X}^{\prime}=0$ for all $\mathbb{X}$ then $\mathbb{X}^{\prime}=0$. In order to expand the action (1.2), one decomposes

$$
\mathbb{A}=\mathbb{A}_{(L)} \star \frac{1}{2}(1+\gamma)+\mathbb{A}_{(R)} \star \frac{1}{2}(1-\gamma),
$$

where $\mathbb{A}_{(c)}(c=L, R)$ are $\gamma$-independent. The action, which is thus the natural fractional spin generalization of chirally asymmetric Chern-Simons (super)gravities [17, 18], thus takes the form

$$
\begin{aligned}
S_{ \pm}^{(x)}[\mathbb{A}] & =\frac{\varkappa}{2 \pi} \int_{M_{3}} \operatorname{Tr}_{\mathcal{A}_{ \pm}}^{(x)}\left[\frac{1}{2} \mathbb{A} \star d \mathbb{A}+\frac{1}{3} \mathbb{A} \star \mathbb{A} \star \mathbb{A}\right] \\
& =\frac{1+x}{2} S_{ \pm}\left[\mathbb{A}_{(L)}\right]-\frac{1-x}{2} S_{ \pm}\left[\mathbb{A}_{(R)}\right]
\end{aligned}
$$

where the chiral action $(c=L, R)$

$$
S_{ \pm}\left[\mathbb{A}_{(c)}\right]=\frac{\varkappa}{2 \pi} \int_{M_{3}}\left[\mathcal{L}_{\mathrm{CS}}\left(W_{(c)}\right) \pm \mathcal{L}_{\mathrm{CS}}\left(U_{(c)}\right)+\frac{1}{2} \mathrm{STr}_{\overline{A q}(2 ; \nu)}\left(\psi_{(c)} \star D \bar{\psi}_{(c)} \pm \bar{\psi}_{(c)} \star D \psi_{(c)}\right)\right]
$$

is defined in terms of the Chern-Simons Lagrangian

$$
\mathcal{L}_{\mathrm{CS}}\left(W_{(c)}\right)=\operatorname{STr}_{\overline{A q}(2 ; \nu)}\left[\frac{1}{2} W_{(c)} \star d W_{(c)}+\frac{1}{3} W_{(c)} \star W_{(c)} \star W_{(c)}\right],
$$

idem $\mathcal{L}_{\mathrm{CS}}\left(U_{(c)}\right)$ and the covariant derivatives

$$
D \psi_{(c)}=d \psi_{(c)}+W_{(c)} \star \psi_{(c)}+\psi_{(c)} \star U_{(c)}, \quad D \bar{\psi}_{(c)}=d \bar{\psi}_{(c)}+U_{(c)} \star \bar{\psi}_{(c)}+\bar{\psi}_{(c)} \star W_{(c)} .
$$

\section{Construction of quasi-projectors}

In this section we construct the non-polynomial elements, or Wigner distributions, in the fractional spin algebra. We shall show that they form an associative and traceable algebra provided that the square of a certain ground state quasi-projector is finite (see eq. (3.23)), which is the main hypothesis underlying our construction, and that we hope to demonstrate fully elsewhere. In the present paper, we show its validity to the first 2 orders in an expansion variable, and each time, to all orders in $\nu$. 


\subsection{Creation and annihilation operator basis}

To construct the quasi-projectors it is convenient to change from the Lorentz covariant basis (2.3) to a basis of $O(2)_{J_{0}}$ covariant deformed creation and annihilation operators

$$
a^{ \pm}=u^{ \pm \alpha} q_{\alpha}, \quad u^{+\alpha} u_{\alpha}^{-}=-\frac{i}{2}, \quad\left(u_{\alpha}^{ \pm}\right)^{\dagger}=u_{\alpha}^{\mp} .
$$

These operators obey

$$
\left[a^{-}, a^{+}\right]_{\star}=1+\nu k, \quad\left\{k, a^{ \pm}\right\}_{\star}=0, \quad\left(a^{ \pm}\right)^{\dagger}=a^{\mp},
$$

from which follows the contraction rules

$$
\begin{aligned}
a^{ \pm} \star\left[\left(a^{\mp}\right)^{m}\left(a^{ \pm}\right)^{n}\right]^{\sigma, \sigma^{\prime}}= & {\left[\left(a^{\mp}\right)^{m}\left(a^{ \pm}\right)^{n+1}\right]^{-\sigma, \sigma^{\prime}} } \\
& \mp \frac{m}{2}\left(1-\frac{m+n+\frac{1}{2}\left(1-(-1)^{m+n}\right)}{(m+n)(m+n+1)} \nu \sigma\right)\left[\left(a^{\mp}\right)^{m-1}\left(a^{ \pm}\right)^{n}\right]^{-\sigma, \sigma^{\prime}}, \\
{\left[\left(a^{\mp}\right)^{m}\left(a^{ \pm}\right)^{n}\right]^{\sigma, \sigma^{\prime}} \star a^{ \pm}=} & {\left[\left(a^{\mp}\right)^{m}\left(a^{ \pm}\right)^{n+1}\right]^{\sigma,-\sigma^{\prime}} } \\
& \pm \frac{m}{2}\left(1-\frac{m+n+\frac{1}{2}\left(1-(-1)^{m+n}\right)}{(m+n)(m+n+1)} \nu \sigma\right)\left[\left(a^{\mp}\right)^{m-1}\left(a^{ \pm}\right)^{n}\right]^{\sigma,-\sigma^{\prime}},
\end{aligned}
$$

where

$$
\left[\left(a^{+}\right)^{m}\left(a^{-}\right)^{n}\right]^{\sigma, \sigma^{\prime}} \equiv\left(u^{+\alpha}\right)^{m}\left(u^{-\alpha}\right)^{n} T_{\alpha(m+n)}^{\sigma, \sigma^{\prime}},
$$

using a shorthand notation in which $\left(u^{ \pm \alpha}\right)^{m}=u^{ \pm \alpha_{1}} \cdots u^{ \pm \alpha_{m}}$. In this basis, the spin operator (2.23) and the basic commutation rules involving it and the deformed oscillators take the form

$$
w=a^{+} a^{-}=\frac{1}{2}\left\{a^{-}, a^{+}\right\}_{\star}, \quad\left[w, a^{ \pm}\right]_{\star}= \pm a^{ \pm} .
$$

Writing $w^{m}=\left(a^{+}\right)^{m}\left(a^{-}\right)^{m}$, one also has the useful relations

$$
a^{ \pm} \star\left[w^{m}\right]^{\sigma, \sigma}=\left[a^{ \pm}\left(w^{m} \mp \frac{m(2 m+1-\nu \sigma)}{2(2 m+1)} w^{m-1}\right)\right]^{-\sigma, \sigma},
$$

and

$$
\begin{aligned}
w \star\left[w^{m}\right]^{\sigma, \sigma} & =\left[w^{m+1}+\lambda_{m}^{\sigma} w^{m-1}\right]^{\sigma, \sigma}, \\
a^{\epsilon} \star\left[w^{m}\right]^{\sigma, \sigma} \star a^{-\epsilon} & =\left[w^{m+1}+\epsilon \mu_{m}^{\sigma} w^{m}+\tilde{\lambda}_{m}^{\sigma} w^{m-1}\right]^{-\sigma,-\sigma},
\end{aligned}
$$

where we have defined

$$
\begin{aligned}
\lambda_{m}^{\sigma} & =-\frac{m^{2}}{4} \frac{(2 m+1-\nu \sigma)(2 m-1+\nu \sigma)}{(2 m+1)(2 m-1)}, \\
\mu_{m}^{\sigma}=-\frac{1}{2}(2 m+1-\nu \sigma), \quad \tilde{\lambda}_{m}^{\sigma} & =\frac{m^{2}}{4} \frac{(2 m+1-\nu \sigma)(2 m-1-\nu \sigma)}{(2 m+1)(2 m-1)} .
\end{aligned}
$$




\subsection{Non-critical versus critical $\nu$}

From (3.8) and (3.10) it follows that if

$$
\nu \notin 2 \mathbb{Z}+1 \quad(\text { non-critical } \nu),
$$

then all eigenvalues of $w$ are non-degenerate and all quasi-projectors are descendants of the ground state quasi-projectors

$$
T_{\epsilon}^{\sigma} \equiv T_{\lambda_{\epsilon, 0}^{\sigma}|\epsilon| \lambda_{\epsilon, 0}^{\sigma}}^{\sigma, \sigma}
$$

obeying

$$
a^{-\epsilon} \star T_{\epsilon}^{\sigma}=0, \quad \lambda_{\epsilon, 0}^{\sigma}=\frac{\epsilon(1+\nu \sigma)}{2},
$$

which makes $T_{\epsilon}^{\sigma}$ into a lowest (highest) weight state for $\epsilon=+(\epsilon=-)$. The resulting spectrum of generalized quasi-projectors and corresponding eigenvalues is given by

$$
\begin{aligned}
T_{\lambda_{\epsilon, m}^{\sigma}|\epsilon| \lambda_{\epsilon, n}^{\sigma}}^{\sigma_{m}, \sigma_{n}} & :=\left(a^{\epsilon}\right)^{\star m} \star T_{\epsilon}^{\sigma} \star\left(a^{-\epsilon}\right)^{\star n}, & & m, n \in\{0,1,2, \ldots\}, \\
\lambda_{\epsilon, m}^{\sigma} & =\epsilon\left(m+\frac{1}{2}(1+\nu \sigma)\right), & \sigma_{m} & =(-1)^{m} \sigma .
\end{aligned}
$$

However, when

$$
\nu \in 2 \mathbb{Z}+1 \quad(\text { critical } \nu),
$$

then the spectrum degenerates, as illustrated in figure 1, and singular quasi-projectors arise, leading to an indecomposable structure to be examined below once we have completed the definition of the model.

\subsection{Projectors in non-critical case}

For non-critical $\nu$, we define the ground state quasi-projectors

$$
T_{\epsilon}^{\sigma}=\sum_{m=0}^{\infty} f_{m} w^{m} \star \Pi^{\sigma}, \quad f_{0}=1
$$

obeying

$$
a^{-\epsilon} \star T_{\epsilon}^{\sigma}=0=T_{\epsilon}^{\sigma} \star a^{\epsilon}, \quad \operatorname{STr}_{\overline{A q}(2 ; \nu)} T_{\epsilon}^{\sigma}=\frac{1}{2}(1-\nu \sigma) .
$$

Using (3.7), one finds a recursive relation for $f_{m}$ with a unique solution with $f_{0}=1$, given by

$$
f_{m}=\frac{(-2 \epsilon)^{m}}{m !} \frac{\left(\frac{3}{2}\right)_{m}}{\left(\frac{3-\nu \sigma}{2}\right)_{m}},
$$

where the Pochhammer symbol $(a)_{n}$ is given by 1 if $n=0$ and by $a(a+1) \cdots(a+n-1)$ if $n=1,2, \ldots$ Hence, using the definition of the confluent hypergeometric function, viz.

$$
{ }_{1} F_{1}(a ; b ; z)=\sum_{n \geqslant 0} \frac{(a)_{n}}{(b)_{n}} \frac{z^{n}}{n !},
$$


we have

$$
T_{\epsilon}^{\sigma}={ }_{1} F_{1}\left(\frac{3}{2} ; \frac{3-\sigma \nu}{2} ;-2 \epsilon w\right) \star \Pi^{\sigma},
$$

which obeys $\left(w-\lambda_{\epsilon, 0}^{\sigma}\right) \star T_{\epsilon}^{\sigma}=0$ by virtue of (3.8).

The rest of our analysis will be based on the assumption that $T_{\epsilon}^{\sigma} \star T_{\epsilon}^{\sigma}$ is finite and non-vanishing, which is equivalent to that

$$
\left.T_{\epsilon}^{\sigma} \star T_{\epsilon}^{\sigma}=\left(\mathcal{N}_{\epsilon}^{\sigma}\right)^{-1} T_{\epsilon}^{\sigma}, \quad \mathcal{N}_{\epsilon}^{\sigma} \in\right] 0, \infty[
$$

in view of the uniqueness of the solution to (3.19). The normalized ground state projector is then defined as follows:

$$
P_{\lambda_{\epsilon, 0}^{\sigma}|\epsilon| \lambda_{\epsilon, 0}^{\sigma}}^{\sigma, \sigma} \equiv P_{\epsilon}^{\sigma}=\mathcal{N}_{\epsilon}^{\sigma} T_{\epsilon}^{\sigma}, \quad P_{\epsilon}^{\sigma} \star P_{\epsilon}^{\sigma}=P_{\epsilon}^{\sigma}
$$

In this section, we will prove that (3.23) holds true to zeroth and first order in the variable $w$, and this, to all orders in $\nu$. That the equation (3.23) is true to all orders in $w$ can be proven by a direct, though tedious analysis that we will present elsewhere.

Let us first prove (3.23) to zeroth order in $w$. The normalization can be obtained from

$$
\left(\mathcal{N}_{\epsilon}^{\sigma}\right)^{2} \operatorname{STr}_{\overline{A q}(2 ; \nu)}\left(T_{\epsilon}^{\sigma} \star T_{\epsilon}^{\sigma}\right)=\mathcal{N}_{\epsilon}^{\sigma} \operatorname{STr}_{\overline{A q}(2 ; \nu)} T_{\epsilon}^{\sigma},
$$

using

$$
\mathrm{STr}_{\overline{A q}(2 ; \nu)} T_{\epsilon}^{\sigma}=\mathrm{S}_{\operatorname{Tr}_{\overline{A q}(2 ; \nu)}} \Pi^{\sigma}=\frac{1}{2}(1-\nu \sigma)
$$

and

$$
\mathrm{STr}_{\overline{A q}(2 ; \nu)}\left(T_{\epsilon}^{\sigma} \star T_{\epsilon}^{\sigma}\right)=\sum_{m, n} f_{m} f_{n} \mathrm{STr}_{A q(2 ; \nu)}\left(w^{m} \star w^{n} \star \Pi^{\sigma}\right),
$$

where

$$
\operatorname{STr}_{A q(2 ; \nu)}\left(w^{m} \star w^{n} \star \Pi^{\sigma}\right)=\delta_{m, n} \operatorname{STr}_{A q(2 ; \nu)}\left(w^{m} \star w^{m} \star \Pi^{\sigma}\right),
$$

from which it follows that

$$
\operatorname{STr}_{A q(2 ; \nu)}\left(w^{m} \star w^{m} \star \Pi^{\sigma}\right)=\operatorname{STr}_{A q(2 ; \nu)}(\underbrace{w \star \cdots \star w}_{m \text { times }} \star w^{m} \star \Pi^{\sigma})=\frac{1}{2}(1-\sigma \nu) \prod_{n=1}^{m} \lambda_{n}^{\sigma},
$$

where we have used (3.8) and (3.10). Hence,

$$
\begin{aligned}
\left(\mathcal{N}_{\epsilon}^{\sigma}\right)^{-1} & =\sum_{m=0}^{\infty}\left(f_{m}\right)^{2} \prod_{n=1}^{m} \lambda_{n}^{\sigma} \\
& =2{ }_{2} F_{1}\left(\frac{1+\nu \sigma}{2}, 2 ; \frac{3-\nu \sigma}{2} ;-1\right)-{ }_{2} F_{1}\left(\frac{1+\nu \sigma}{2}, 1 ; \frac{3-\nu \sigma}{2} ;-1\right) \\
& =2{ }_{2} F_{1}\left(\frac{3+\nu \sigma}{2}, 2 ; \frac{3-\nu \sigma}{2} ;-1\right) \\
& =\frac{1}{2}(1-\nu \sigma),
\end{aligned}
$$


where we have used Gauss' contiguous relations between hypergeometric functions followed by Kummer's evaluation formula.

In order to prove (3.23) to the first order in $w$, we can use the structure constants of the lone-star product [19] (see also [20]) in the form ${ }^{9}$

$$
w^{m} \star w^{n}=\sum_{p=0}^{2 m} C_{p}^{(m, n)} w^{m+n-p}
$$

where

$$
\begin{aligned}
C_{p}^{(m, n)} & =\left(\frac{1}{2}\right)^{p} \frac{1}{p !} \mathcal{N}_{p}^{m, n} \phi_{p}^{(m, n)}(\nu), \\
\mathcal{N}_{p}^{m, n} & =\sum_{r=0}^{p}(-1)^{r}\left(\begin{array}{l}
p \\
r
\end{array}\right)[m]_{r}[m]_{p-r}[n]_{r}[n]_{p-r},
\end{aligned}
$$

and

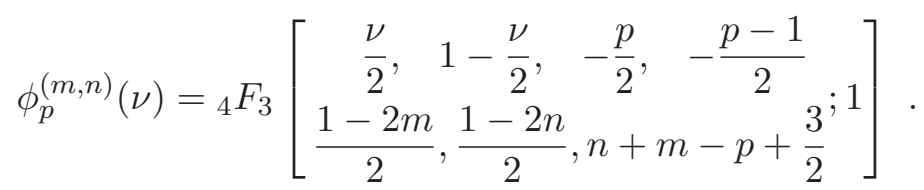

Defining the operator $\Delta(\cdot):=\left.\frac{d(\cdot)}{d w}\right|_{w=0}$, to first order in $w$ we have

$$
\Delta\left(T_{\epsilon}^{\sigma} \star T_{\epsilon}^{\sigma}\right)=2 \sum_{m=0}^{\infty} f_{m+1} f_{m} C_{2 m}^{(m, m+1)},
$$

giving, after some algebra,

$$
\begin{aligned}
\Delta\left(T_{\epsilon}^{\sigma} \star T_{\epsilon}^{\sigma}\right) & =2 \sum_{m=0}^{\infty} \frac{(-2 \epsilon)^{m+1}\left(\frac{3}{2}\right)_{m+1}}{(m+1) !\left(\frac{3-\sigma \nu}{2}\right)_{m+1}} \frac{(-2 \epsilon)^{m}\left(\frac{3}{2}\right)_{m}}{m !\left(\frac{3-\sigma \nu}{2}\right)_{m}}\left(\frac{1}{2}\right)^{2 m}(-1)^{m}(2)_{m}^{2} \frac{\left(\frac{5-\nu}{2}\right)_{m}\left(\frac{3+\nu}{2}\right)_{m}}{\left(\frac{5}{2}\right)_{m}\left(\frac{3}{2}\right)_{m}} \\
& =2 \frac{(-2 \epsilon) \frac{3}{2}}{\frac{3-\sigma \nu}{2}} \sum_{m=0}^{\infty} \frac{(2)_{m}\left(\frac{5-\nu}{2}\right)_{m}\left(\frac{3+\nu}{2}\right)_{m}}{\left(\frac{5-\sigma \nu}{2}\right)_{m} m !\left(\frac{3-\sigma \nu}{2}\right)_{m}}(-1)^{m}
\end{aligned}
$$

Taking $\sigma=+1$ for the sake of definiteness and without loss of generality (as we can always revert the sign of $\nu$ in the final result), we obtain

$$
\begin{aligned}
\Delta\left(T_{\epsilon}^{+} \star T_{\epsilon}^{+}\right) & =2 f_{1} \sum_{m=0}^{\infty} \frac{(2)_{m}\left(\frac{3+\nu}{2}\right)_{m}}{m !\left(\frac{3-\nu}{2}\right)_{m}}(-1)^{m}=2 f_{1}{ }_{2} F_{1}\left[\frac{3+\nu}{2}, 2 ; \frac{3-\nu}{2} ;-1\right], \\
\Longrightarrow \Delta\left(T_{\epsilon}^{+} \star T_{\epsilon}^{+}\right) & =\frac{1-\nu}{2} f_{1},
\end{aligned}
$$

where we used Kummer's theorem to evaluate the hypergeometric function. We have thus proven the correctness of (3.23) to zeroth and first orders in $w$, and each time, to all orders in $\nu$.

\footnotetext{
${ }^{9}$ The result can also be obtained by brute force [21, 22].
} 
Thus, for non-critical $\nu$ and under the assumption made on (3.23), the ground state projectors are given by

$$
P_{\epsilon}^{\sigma}=\frac{2}{1-\nu \sigma}{ }_{1} F_{1}\left(\frac{3}{2} ; \frac{3-\sigma \nu}{2} ;-2 \epsilon w\right) \star \Pi^{\sigma} .
$$

Their supertraces are given by ${ }^{10}$

$$
\mathrm{STr}_{\overline{A q}(2 ; \nu)} P_{\epsilon \lambda_{0} \mid \epsilon \lambda_{0}}^{\sigma, \sigma}=1 .
$$

From these ground state projectors descend matrices of generalized projectors

$$
\left(P_{\epsilon}^{\sigma_{m}, \sigma_{n}}\right)_{\lambda_{\epsilon, m}^{\sigma}} \lambda_{\epsilon, n}^{\sigma}=\frac{2}{1-\nu \sigma} \mathcal{C}_{\epsilon, m}^{\sigma} \mathcal{N}_{\epsilon, m}^{\sigma} \mathcal{N}_{\epsilon, n}^{\sigma} T_{\lambda_{\epsilon, m}^{\sigma}|\epsilon| \lambda_{\epsilon, n}^{\sigma}}^{\sigma_{m}, \sigma_{n}},
$$

where the normalization and conjugation coefficients are given by

$$
\mathcal{N}_{\epsilon, m}^{\sigma}=\left|\eta_{\epsilon, m}^{\sigma}\right|^{-\frac{1}{2}}, \quad \mathcal{C}_{\epsilon, m}^{\sigma}=\frac{\eta_{\epsilon, m}^{\sigma}}{\left|\eta_{\epsilon, m}^{\sigma}\right|},
$$

respectively, where the coefficients

$$
\left(a^{-\epsilon}\right)^{\star m} \star\left(a^{\epsilon}\right)^{\star m} \star T_{\epsilon}^{\sigma}=\eta_{\epsilon, m}^{\sigma} T_{\epsilon}^{\sigma} .
$$

More explicitly, we have $\eta_{\epsilon, 0}^{\sigma}=1$ and

$$
\eta_{\epsilon, m}^{\sigma}=\epsilon^{m} \prod_{n=1}^{m}\left(n+\frac{1}{2}\left(1-(-1)^{n}\right) \sigma \nu\right) \quad \text { for } m=1,2, \ldots .
$$

The usage of the conjugation coefficients in (3.43) implies the reality condition

$$
\left(\left(P_{\epsilon}^{\sigma, \sigma^{\prime}}\right)_{\lambda} \lambda^{\prime}\right)^{\dagger}=C \star\left(P_{\epsilon}^{\sigma^{\prime}, \sigma}\right)_{\lambda^{\prime}}^{\lambda} \star C,
$$

where the conjugation matrix $[8,16]$

$$
C=\sum_{m, \epsilon, \sigma} \mathcal{C}_{\epsilon, m}^{\sigma}\left(P_{\epsilon}^{\sigma_{m}, \sigma_{m}}\right)_{\lambda_{\epsilon, m}^{\sigma}} \lambda_{\epsilon, m}^{\sigma} .
$$

Turning to the supertrace of the generalized projectors, they are given by

$$
\begin{aligned}
\operatorname{STr}_{\overline{A q}(2 ; \nu)}\left[P_{\epsilon}^{\sigma_{m}, \sigma_{n}}\right]_{\lambda_{\epsilon, m}^{\sigma}} \lambda_{\epsilon, n}^{\sigma}=\frac{2 \mathcal{C}_{\epsilon, m}^{\sigma} \mathcal{N}_{\epsilon, m}^{\sigma} \mathcal{N}_{\epsilon, n}^{\sigma}}{1-\nu \sigma} \operatorname{STr}_{\overline{A q}(2 ; \nu)}\left[\left(a^{\epsilon}\right)^{\star m} \star T_{\epsilon}^{\sigma} \star\left(a^{-\epsilon}\right)^{\star m}\right] \\
=\frac{2 \mathcal{C}_{\epsilon, m}^{\sigma} \mathcal{N}_{\epsilon, m}^{\sigma} \mathcal{N}_{\epsilon, n}^{\sigma} \operatorname{STr}_{\overline{A q}(2 ; \nu)}\left[\left(a^{\epsilon}\right)^{\star m} \star \sum_{n=0}^{m} f_{n} w^{n} \star\left(a^{-\epsilon}\right)^{\star m}\right]}{1-\nu \sigma}\left[\frac{2(-1)^{m} \mathcal{C}_{\epsilon, m}^{\sigma} \mathcal{N}_{\epsilon, m}^{\sigma} \mathcal{N}_{\epsilon, n}^{\sigma} \operatorname{STr}_{\overline{A q}(2 ; \nu)}\left[\left(a^{-\epsilon}\right)^{\star m} \star\left(a^{\epsilon}\right)^{\star m} \star \sum_{n=0}^{m} f_{n} w^{n}\right]}{1-\nu \sigma}=\frac{2(-1)^{m} \mathcal{C}_{\epsilon, m}^{\sigma} \mathcal{N}_{\epsilon, m}^{\sigma} \mathcal{N}_{\epsilon, n}^{\sigma} \operatorname{STr}_{\overline{A q}(2 ; \nu)}\left[\left(a^{-\epsilon}\right)^{\star m} \star\left(a^{\epsilon}\right)^{\star m} \star T_{\epsilon}^{\sigma}\right]}{1-\nu \sigma}\right.
\end{aligned}
$$

\footnotetext{
${ }^{10}$ The $\nu$-independence of (3.42) (to be compared with the remark below eq. (2.41) in [8]) is in accordance with realizing $\left(a^{ \pm}, k\right)$ using undeformed oscillators $b^{ \pm}$obeying $\left[b^{-}, b^{+}\right]_{\star}=1[8,16]$. The vacuum projectors $P_{\epsilon}^{\sigma}$ can then be represented in $\overline{A q}(2 ; 0)$ by $2 \exp \left(-2 \epsilon b^{+} b^{-}\right) \star \Pi^{\sigma}$ with $\operatorname{STr}_{\overline{A q}(2 ; 0)}\left(2 \exp \left(-2 \epsilon b^{+} b^{-}\right) \star \Pi^{\sigma}\right)=1$.
} 
where we have allowed ourselves to exchange the order of sums and supertraces and used

$$
\operatorname{STr}_{\overline{A q}(2 ; \nu)}\left(\left(a^{\epsilon}\right)^{\star m} \star w^{n} \star\left(a^{-\epsilon}\right)^{\star m}\right)=0=\mathrm{S}_{\overline{A q}(2 ; \nu)}\left(\left(a^{-\epsilon}\right)^{\star m} \star\left(a^{\epsilon}\right)^{\star m} \star w^{n}\right) \quad n>m,
$$

and the graded cyclic property of the supertrace operation in the algebra of polynomials. Thus, it follows from (3.44) and (3.45) that

$$
\operatorname{STr}_{\overline{A q}(2 ; \nu)}\left(P_{\epsilon}^{\sigma_{m}, \sigma_{n}}\right)_{\lambda_{\epsilon, m}^{\sigma}} \lambda_{\epsilon, n}^{\sigma}=(-1)^{m} \delta_{m n},
$$

as one can indeed verify explicitly by making repeated use of (3.9), (3.44) and (3.46). Finally, under the assumption that (3.23) holds true, it follows from (3.43) that

$$
\left(P_{\epsilon}^{\sigma, \sigma^{\prime}}\right)_{\lambda}^{\lambda^{\prime}} \star\left(P_{\epsilon}^{\sigma^{\prime \prime}, \sigma^{\prime \prime \prime}}\right)_{\lambda^{\prime \prime}} \lambda^{\prime \prime \prime}=\delta^{\sigma^{\prime} \sigma^{\prime \prime}} \delta_{\lambda^{\prime \prime}}^{\lambda^{\prime}}\left(P_{\epsilon}^{\sigma, \sigma^{\prime \prime \prime}}\right)_{\lambda} \lambda^{\prime \prime \prime}
$$

which in its turn implies the graded cyclic property of the supertrace operation.

In summary so far, the salient features of the fractional spin algebra, namely its associativity and traceability using the extension of Vasiliev's supertrace, thus hinge on the finiteness of the normalization coefficient $\mathcal{N}_{\epsilon}^{\sigma}$ in (3.23), which we hope to demonstrate in a forthcoming work by using a convolution formula for the star product in $A q(2 ; \nu)$. Since we have proven the correctness of (3.23) to the first 2 orders in $w$, we believe it holds true to all orders. ${ }^{11}$

\subsection{Quasi-projectors for critical $\nu= \pm 3, \pm 5, \ldots$}

Turning to critical $\nu$, we first consider the case

$$
\nu=\hat{\sigma}(2 \ell+1), \quad \ell=1,2,3, \ldots, \quad \hat{\sigma}= \pm 1,
$$

leaving the hyper-critical case $\nu= \pm 1$ to the end. Letting $\widehat{T}_{\epsilon}^{\sigma}$ denote the corresponding critical ground state quasi-projectors in $\overline{A q}(2 ; \nu)$, i.e.

$$
a^{-\epsilon} \star \widehat{T}_{\epsilon}^{\sigma}=0, \quad\left(w-\lambda_{\epsilon, 0}^{\sigma}\right) \star \widehat{T}_{\epsilon}^{\sigma}=0, \quad \lambda_{\epsilon, 0}^{-\hat{\sigma}}=\epsilon \ell, \quad \lambda_{\epsilon, 0}^{\hat{\sigma}}=\epsilon(\ell+1),
$$

we can take

$$
\widehat{T}_{\epsilon}^{-\hat{\sigma}}=\lim _{\nu \rightarrow(2 \ell+1) \hat{\sigma}}{ }_{1} F_{1}\left(\frac{3}{2} ; \frac{3+\hat{\sigma} \nu}{2} ;-2 \epsilon w\right) \star \Pi^{-\hat{\sigma}}={ }_{1} F_{1}\left(\frac{3}{2} ; \ell+2 ;-2 \epsilon w\right) \star \Pi^{-\hat{\sigma}},
$$

which is a non-singular quasi-projector leading to the projector

$$
\widehat{P}_{\epsilon}^{-\hat{\sigma}}=\frac{1}{\ell+1}{ }_{1} F_{1}\left(\frac{3}{2} ; \ell+2 ;-2 \epsilon w\right) \star \Pi^{-\hat{\sigma}} .
$$

On the other hand, from

$$
\frac{\nu-(2 \ell+1) \hat{\sigma}}{\left(\frac{3-\hat{\sigma} \nu}{2}\right)_{n}} \rightarrow \begin{cases}0 & \text { for } n \leqslant \ell-1, \\ \frac{2(-1)^{\ell} \hat{\sigma}}{(\ell-1) !(n-l) !} & \text { for } n \geqslant \ell,\end{cases}
$$

\footnotetext{
${ }^{11}$ Using the basic star product formula to evaluate the left-hand side of (3.23), we expect the coefficient of $w^{m}$ to be a sum of ${ }_{p} F_{q}(\cdots ; \cdots ; z)$ functions evaluated at $z=-1$.
} 
and

$$
\left(\frac{3}{2}\right)_{n}=\left(\frac{3}{2}\right)_{\ell}\left(\frac{3}{2}+\ell\right)_{n-\ell}, \quad n \geqslant \ell,
$$

it follows that

$$
\lim _{\nu \rightarrow(2 \ell+1) \hat{\sigma}}(\nu-(2 \ell+1) \hat{\sigma}){ }_{1} F_{1}\left(\frac{3}{2} ; \frac{3-\hat{\sigma} \nu}{2} ;-2 \epsilon w\right) \star \Pi^{\hat{\sigma}}=\frac{2^{\ell+1} \epsilon^{\ell} \hat{\sigma}\left(\frac{3}{2}\right)_{\ell}}{\ell !(\ell-1) !} \widehat{T}_{\epsilon}^{\hat{\sigma}},
$$

where we have defined

$$
\widehat{T}_{\epsilon}^{\hat{\sigma}}=\left[w^{\ell}{ }_{1} F_{1}\left(\ell+\frac{3}{2} ; \ell+1 ;-2 \epsilon w\right)\right]^{\hat{\sigma}, \hat{\sigma}} .
$$

This element obeys

$$
a^{-\epsilon} \star \widehat{T}_{\epsilon}^{\hat{\sigma}}=0, \quad(w-\epsilon(\ell+1)) \star \widehat{T}_{\epsilon}^{\hat{\sigma}}=0,
$$

which can indeed be checked using (3.8). Moreover, by taking the limit of the normalization condition (3.25), under the assumption that $\widehat{T}_{\epsilon}^{\hat{\sigma}}$ is supertraceable, one finds

$$
\widehat{T}_{\epsilon}^{\hat{\sigma}} \star \widehat{T}_{\epsilon}^{\hat{\sigma}}=0,
$$

that we refer to as $\widehat{T}_{\epsilon}^{\hat{\sigma}}$ being a singular quasi-projector, which can hence not be normalized to form a projector. To analyze descendants, we first use (3.8) and (3.10) to show that if

$$
\widehat{T}_{\lambda ; p}^{\sigma}=\sum_{m=p}^{\infty} f_{m}^{\sigma} w^{m} \star \Pi^{\sigma}, \quad(w-\lambda) \star \widehat{T}_{\lambda ; p}^{\sigma}=0, \quad f_{p}^{\sigma}=1,
$$

then one has one of the following cases:

$$
\begin{array}{ll}
(p, \sigma)=(0, \hat{\sigma}), \quad \lambda \in\left\{ \pm(\ell-1), \pm(\ell-3), \cdots, \pm \frac{1}{2}\left(1+(-1)^{\ell}\right)\right\}, \\
(p, \sigma)=(0,-\hat{\sigma}), \quad \lambda \in\left\{ \pm \ell, \pm(\ell-2), \cdots, \pm \frac{1}{2}\left(1-(-1)^{\ell}\right)\right\}, \\
(p, \sigma)=(\ell, \hat{\sigma}) \quad \text { or } \quad(\ell+1,-\hat{\sigma}) \quad(\text { no condition on } \lambda),
\end{array}
$$

and $\widehat{T}_{\lambda ; \ell}^{\hat{\sigma}}$ and $\widehat{T}_{\lambda ; \ell+1}^{-\hat{\sigma}}$ are unique while $\widehat{T}_{\lambda ; 0}^{\sigma}$ belongs to a two-dimensional solution space. We note that the first case in (3.66) corresponds to the singular ground state quasiprojector, viz.

$$
\widehat{T}_{\epsilon(\ell+1) ; \ell}^{\hat{\sigma}}=\widehat{T}_{\epsilon}^{\hat{\sigma}} .
$$

The quantization of $\lambda$ in eqs. (3.64) and (3.65) follows from the fact that $\left\{f_{m}^{\hat{\sigma}}\right\}_{m=0}^{\ell-1}$ and $\left\{f_{m}^{-\hat{\sigma}}\right\}_{m=0}^{\ell}$ must obey the homogenous equation systems

$$
\begin{aligned}
f_{m-1}^{\hat{\sigma}}-\lambda f_{m}^{\hat{\sigma}}-\lambda_{m+1}^{\hat{\sigma}} f_{m+1}=0, & \lambda_{m}^{\hat{\sigma}}=-\frac{m^{2}\left(m^{2}-\ell^{2}\right)}{4 m^{2}-1} \equiv \lambda_{m}(\ell), \\
f_{m-1}^{-\hat{\sigma}}-\lambda f_{m}^{-\hat{\sigma}}-\lambda_{m+1}^{-\hat{\sigma}} f_{m+1}=0, & \lambda_{m}^{-\hat{\sigma}}=\lambda_{m}(\ell+1),
\end{aligned}
$$


which have rank $r=\ell+\frac{1}{2}(1-\hat{\sigma})$, leading to the characteristic equations

$$
\operatorname{det}\left[\begin{array}{ccccccccc}
\lambda & -\lambda_{1}(r) & 0 & \ldots & & & & & \\
-1 & \lambda & -\lambda_{2}(r) & 0 & \ldots & & & & \\
0 & -1 & \lambda & -\lambda_{3}(r) & 0 & \ldots & & & \\
\vdots & 0 & -1 & \ddots & \ddots & \ddots & & & \\
& \vdots & 0 & \ddots & & & & & \\
& & \vdots & \ddots & & & \ddots & \ddots & \\
& & & & & & \ddots & \lambda & -\lambda_{r-1}(r) \\
& & & & & & & -1 & \lambda
\end{array}\right]=0 .
$$

Thus, descending from the non-singular quasi-projector one encounters an $(2 \ell+1)$-plet of quasi-projectors

$$
\widehat{T}_{\epsilon(m-\ell)|\epsilon| \epsilon(m-\ell)}^{-\hat{\sigma}(-1)^{m},-\hat{\sigma}(-1)^{m}}=\left(a^{+\epsilon}\right)^{\star m} \star \widehat{T}_{\epsilon}^{-\hat{\sigma}} \star\left(a^{-\epsilon}\right)^{\star m}, \quad m=0,1, \ldots, 2 \ell,
$$

all of which have finite supertraces, that is, the element $\widehat{T}_{\epsilon(m-\ell)|\epsilon| \epsilon(m-\ell)}^{-\hat{\sigma}_{m},-\hat{\sigma}_{m}}$ contains a nontrivial component along $\widehat{T}_{\epsilon(m-\ell), 0}^{-\hat{\sigma}_{m}}$. One also has

$$
\tau\left(\widehat{T}_{\epsilon(m-\ell)|\epsilon| \epsilon(m-\ell)}^{-\hat{\sigma}_{m},-\hat{\sigma}_{m}}\right)=\widehat{T}_{-\epsilon(m-\ell)|-\epsilon|-\epsilon(m-\ell)}^{-\hat{\sigma}_{m},-\hat{\sigma}_{m}} .
$$

Descending one step beyond $\widehat{T}_{\epsilon \ell|\epsilon| \epsilon \ell}^{-\hat{\sigma},-\hat{\sigma}}$ one reaches the $\widehat{T}_{\epsilon(\ell+1)|\epsilon| \epsilon(\ell+1)}^{\hat{\sigma}, \hat{\sigma}}$ which is proportional to the singular projector $\widehat{T}_{\epsilon}^{\hat{\sigma}}$, i.e. there exists a non-trivial coefficient $\mathcal{C}_{\ell}^{\hat{\sigma}}$ such that

$$
\widehat{T}_{\epsilon(\ell+1) \mid \epsilon \epsilon \epsilon(\ell+1)}^{\hat{\sigma}, \hat{\sigma}}=\mathcal{C}_{\ell}^{\hat{\sigma}} \widehat{T}_{\epsilon}^{\hat{\sigma}},
$$

which is indeed compatible with the cyclicity of the supertrace. In summary, we have verified necessary conditions for the supertraceability of the algebra $A w(2 ; \nu)$ in the critical cases $\nu= \pm 3, \pm 5, \ldots$. The resulting indecomposable structure, illustrated in figures 2 and 3 , is given by

$$
A w(2 ; \nu)=\frac{A w(2 ; \nu)}{A w^{\prime}(2 ; \nu)} \boxplus A w^{\prime}(2 ; \nu),
$$

where the ideal

$$
A w^{\prime}(2 ; \nu)=\bigoplus_{\epsilon} \bigoplus_{m, n=0}^{\infty}\left[\widehat{T}_{\lambda_{\epsilon, m}|\epsilon| \lambda_{\epsilon, n}^{\hat{\sigma}}}^{(-1)^{m} \hat{\hat{\sigma}},(-1)^{n} \hat{\sigma}} \oplus \widehat{T}_{\lambda_{\epsilon, m+2 \ell+1}^{-}(-1)^{m} \hat{\sigma},(-1)^{n} \hat{\sigma}}^{\left(\lambda_{\epsilon, n+2 \ell+1}\right.}\right]
$$

and hence the coset

$$
\begin{aligned}
\frac{A w(2 ; \nu)}{A w^{\prime}(2 ; \nu)} & =\bigoplus_{\epsilon} \bigoplus_{m, n=0}^{2 \ell} \widehat{T}_{\lambda_{\epsilon, m}^{-\hat{\sigma}}|\epsilon| \lambda_{\epsilon, n}^{-}}^{-(-1)^{m}} \hat{,-(-1)^{n} \hat{\sigma}} \\
& \cong \bigoplus_{\epsilon} g l(2 \ell+1)_{\epsilon} \\
& =\bigoplus_{\epsilon}\left(g l(\ell)^{\hat{\sigma}, \hat{\sigma}} \oplus g l(\ell+1)^{-\hat{\sigma},-\hat{\sigma}} \notin\left[(\ell, \ell+1)^{\hat{\sigma},-\hat{\sigma}} \oplus(\ell+1, \ell)^{-\hat{\sigma}, \hat{\sigma}}\right]\right)_{\epsilon},
\end{aligned}
$$




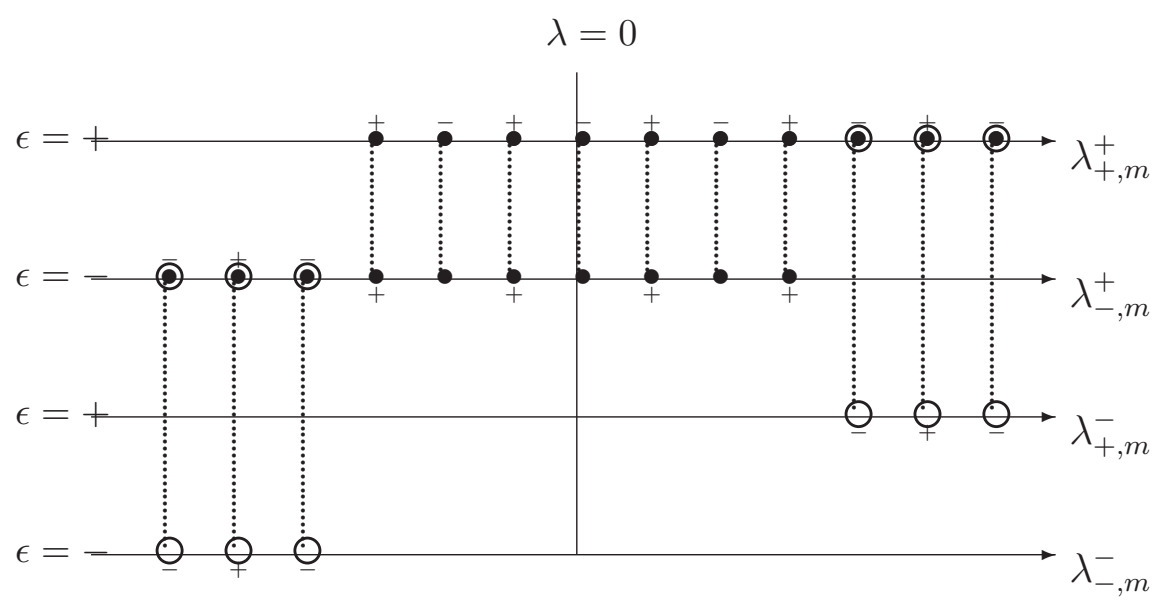

Figure 1. Distribution of the eigenvalues $\lambda_{\epsilon, m}^{\sigma}$ of $w$ for $\ell=3$ and $\hat{\sigma}=-1$. The dashed vertical lines indicate relations between generalized quasi-projectors. In the finite dimensional sector, these elements span two-dimensional solution spaces. In the infinite dimensional sector, these are proportional modulo the rescalings given in (3.59) and (3.73).

which we identify as two copies of the coset $(2.13)$ arising in $A q(2 ; \nu)$ in the critical limit. It is worth mentioning that the appearing of ideal subalgebras in the polynomial basis (2.20) for critical $\nu$ was already noticed in [10], which is also related to the existence of finite dimensional matrix representations of the deformed Heisenberg algebra [16]. Our achievement in this section has been to understand the structure of the algebra of projectors in this critical limit using the tools of symbol calculus.

3.5 Example: $\nu=-3$

If $\nu=-3$, that is, if $\ell=1$ and $\hat{\sigma}=-1$, then the basic contraction formulae read:

$$
\begin{aligned}
w \star\left[w^{m}\right]^{+,+} & =\left[w^{m+1}-\frac{m^{2}(m+2)(m-2)}{(2 m+1)(2 m-1)} w^{m-1}\right]^{+,+}, \\
w \star\left[w^{m}\right]^{-,-} & =\left[w^{m+1}-\frac{m^{2}(m+1)(m-1)}{(2 m+1)(2 m-1)} w^{m-1}\right]^{-,-}, \\
a^{+} \star\left[w^{m}\right]^{+,+} \star a^{-} & =\left[w^{m+1}-(m+2) w^{m}+\frac{m^{2}(m+1)(m+2)}{(2 m+1)(2 m-1)} w^{m-1}\right]^{-,-}, \\
a^{+} \star\left[w^{m}\right]^{-,-} \star a^{-} & =\left[w^{m+1}-(m-1) w^{m}+\frac{m^{2}(m-1)(m-2)}{(2 m+1)(2 m-1)} w^{m-1}\right]^{+,+}
\end{aligned}
$$

The generalized lowest weight state projector

$$
\widehat{T}_{+}^{+}=\left[{ }_{1} F_{1}\left(\frac{3}{2} ; 3 ;-2 w\right)\right]^{+,+}, \quad a^{-} \star \widehat{T}_{+}^{+}=0,(w-1) \star \widehat{T}_{+}^{+}=0 .
$$




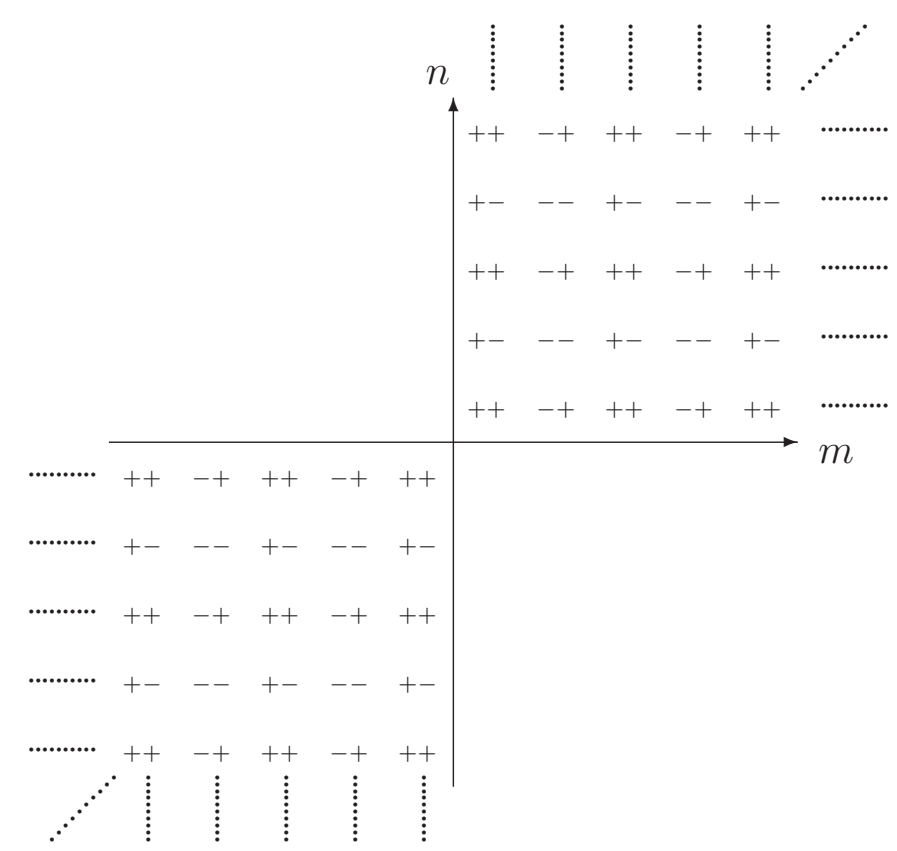

Figure 2. The two sets of singular quasi-projectors $\widehat{T}_{\lambda_{\epsilon, m}^{+}|\epsilon| \lambda_{\epsilon, n}^{+}}^{(-1)^{m}}$ for $\hat{\sigma}=+1$. These are isomorphic to the two sub-ideals drawn in figure 3 .

The descendant quasi-projectors within the triplet representation are given by

$$
\begin{aligned}
& \widehat{T}_{0|+| 0}^{-,-}=a^{+} \star \widehat{T}_{+}^{+} \star a^{-}=-4\left[{ }_{1} F_{1}\left(\frac{3}{2} ; 2 ;-2 w\right)\right]^{-,-}, \\
& \widehat{T}_{1|+| 1}^{+,+}=a^{+} \star T_{0|+| 0}^{-,-} \star a^{-}=-4\left[\left(1-2 w \frac{d}{d w}-w^{2} \frac{d^{2}}{d w^{2}}\right) \widehat{T}_{+}^{+}\right]^{+,+},
\end{aligned}
$$

which indeed have $w$ eigenvalues in accordance with the notation. Descending once more, we find

$$
\widehat{T}_{2|+| 2}^{-,-}=a^{+} \star \widehat{T}_{1|+| 1}^{+,+} \star a^{-}=32 \widehat{T}_{+}^{-},
$$

where the singular quasi-projector

$$
\widehat{T}_{+}^{-}=\left[w_{1} F_{1}\left(\frac{5}{2} ; 2 ;-2 w\right)\right]^{-,-}
$$

One can also check the agreement between computing the supertraces of the above equations either by direct evaluation or by using the graded cyclicity followed by $a^{-} \star a^{+}=$ $w+\frac{1}{2}(1+\nu k)$. 


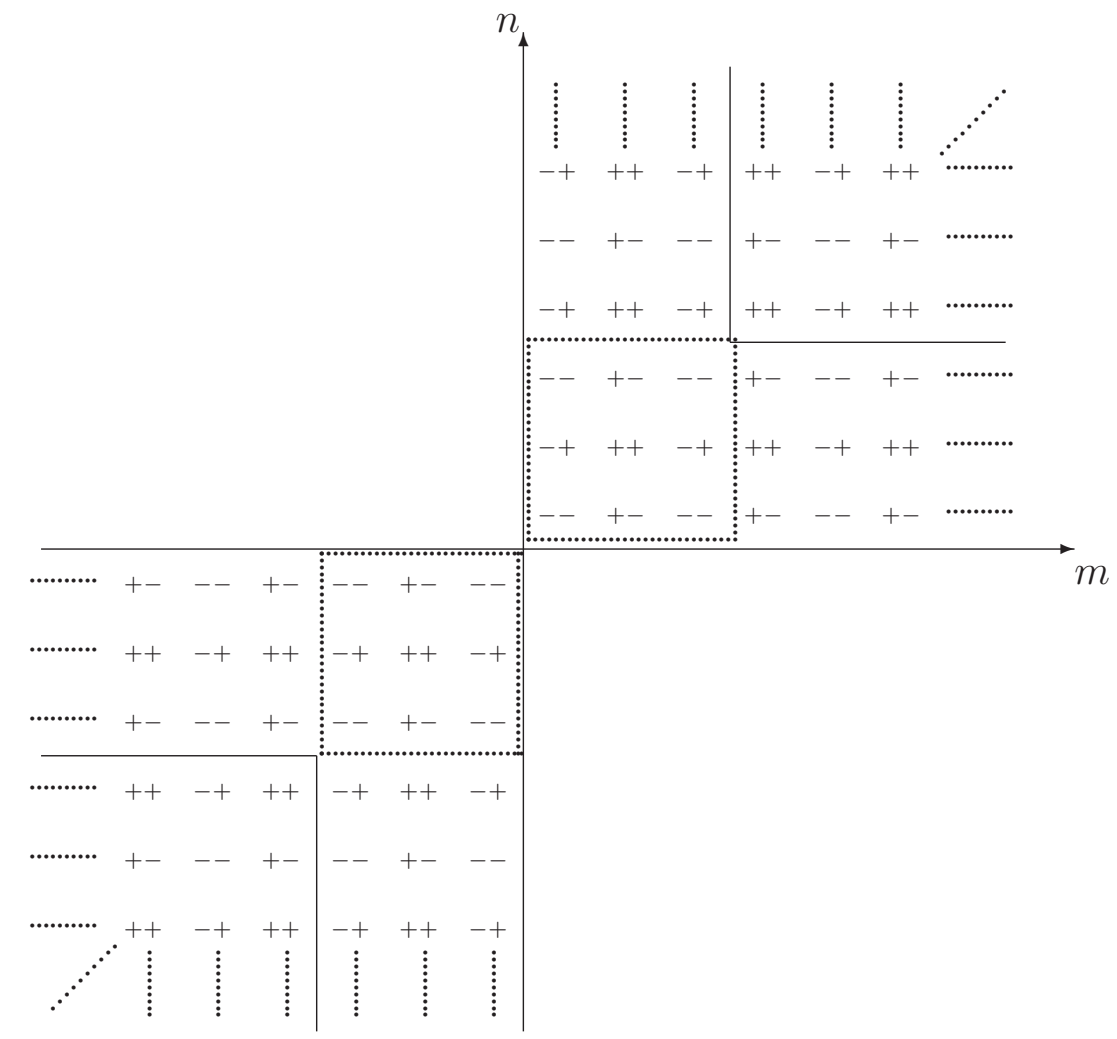

Figure 3. The decomposable structure of the set of quasi-projectors $\widehat{T}_{\lambda_{\epsilon, m}^{-}|\epsilon| \lambda_{\epsilon, n}^{-}}^{-(-1)^{m},-(-1)^{n}}$ for $\hat{\sigma}=+1$ and $\ell=1$. The coset consists of the quasi-projectors encircled by dashed lines. Within the remaining ideal there resides two sub-ideals, separated by solid lines, that are isomorphic to the two sets of singular quasi-projectors depicted in figure 2.

\subsection{The hypercritical cases: $\nu= \pm 1$}

In the hypercritical case we have

$$
\begin{aligned}
\widehat{T}_{\epsilon}^{\hat{\sigma}} & =\lim _{\nu \rightarrow \hat{\sigma}}\left[{ }_{1} F_{1}\left(\frac{3}{2} ; \frac{3-\hat{\sigma} \nu}{2} ;-2 \epsilon w\right)\right]^{\hat{\sigma}, \hat{\sigma}}=\left[{ }_{1} F_{1}\left(\frac{3}{2} ; 1 ;-2 \epsilon w\right)\right]^{\hat{\sigma}, \hat{\sigma}}, \\
\widehat{T}_{\epsilon}^{-\hat{\sigma}} & =\lim _{\nu \rightarrow \hat{\sigma}}\left[{ }_{1} F_{1}\left(\frac{3}{2} ; \frac{3+\hat{\sigma} \nu}{2} ;-2 \epsilon w\right)\right]^{\hat{\sigma}, \hat{\sigma}}=\left[{ }_{1} F_{1}\left(\frac{3}{2} ; 2 ;-2 \epsilon w\right)\right]^{\hat{\sigma}, \hat{\sigma}},
\end{aligned}
$$

with $w$ eigenvalues

$$
w \star \widehat{T}_{\epsilon}^{-\hat{\sigma}}=0, \quad(w-\epsilon) \star \widehat{T}_{\epsilon}^{\hat{\sigma}}=0,
$$

as can be see explicitly using

$$
\begin{aligned}
w \star\left[w^{m}\right]^{\hat{\sigma}, \hat{\sigma}} & =\left[w^{m+1}-\frac{m^{4}}{(2 m+1)(2 m-1)} w^{m-1}\right]^{\hat{\sigma}, \hat{\sigma}}, \\
w \star\left[w^{m}\right]^{-\hat{\sigma},-\hat{\sigma}} & =\left[w^{m+1}-\frac{m^{2}\left(m^{2}-1\right)}{(2 m+1)(2 m-1)} w^{m-1}\right]^{-\hat{\sigma},-\hat{\sigma}} .
\end{aligned}
$$


Using

$$
a^{+\epsilon} \star\left[w^{m}\right]^{-\hat{\sigma},-\hat{\sigma}} \star a^{-\epsilon}=\left[w^{m+1}-\epsilon(m+1) w^{m}+\frac{m^{3}(m+1)}{(2 m+1)(2 m-1)} w^{m-1}\right]^{\hat{\sigma}, \hat{\sigma}},
$$

one can show that

$$
a^{+\epsilon} \star \widehat{T}_{\epsilon}^{-\hat{\sigma}} \star a^{-\epsilon}=-2 \epsilon \widehat{T}_{\epsilon}^{\hat{\sigma}},
$$

which is indeed consistent with supertraceability. Finally, we have

$$
\widehat{P}_{\epsilon}^{-\hat{\sigma}}=\widehat{T}_{\epsilon}^{-\hat{\sigma}}, \quad \widehat{T}_{\epsilon}^{\hat{\sigma}} \star \widehat{T}_{\epsilon}^{\hat{\sigma}}=0,
$$

that is, the element $\widehat{T}_{\epsilon}^{\hat{\sigma}}$ is a singular quasi-projector while $\widehat{T}_{\epsilon}^{-\hat{\sigma}}$ is normalizable.

\section{Final specification of the model}

In this section, we provide the final specifications of the model, in the form of reality conditions and further projections of odd spins. We then compute the resulting relation between the higher spin gravitational and internal gauge couplings.

\subsection{Real forms}

A real form of the model can be obtained by imposing [8]

$$
\mathbb{A}^{\dagger}=-\mathbb{C} \star \mathbb{A} \star \mathbb{C}, \quad \mathbb{C}=\left[\begin{array}{cc}
1 & 0 \\
0 & C \star \Pi^{-}
\end{array}\right],
$$

where the generalized charge conjugation matrix is given by (3.48) and

$$
\gamma^{\dagger}=\gamma, \quad \xi^{\dagger}=\xi
$$

and we assume the standard action of $\dagger$ on $\mathrm{Mat}_{2}$. Thus, in terms of the separate master fields one has

$$
\begin{aligned}
W^{\dagger} & =-W, & U^{\dagger} & =-C \star U \star C, \\
\psi^{\dagger} & =-C \star \bar{\psi}, & \bar{\psi}^{\dagger} & =-\psi \star C .
\end{aligned}
$$

As for the connections, this implies that

$$
W \in \overline{h s}^{+}(\nu)_{(+)} \oplus \overline{h s}^{+}(\nu)_{(-)}, \quad U \in \bigoplus_{\epsilon, \sigma}\left[u(\nu ; \sigma, \epsilon)_{(+)} \oplus u(\nu ; \sigma, \epsilon)_{(-)}\right],
$$

where $\overline{h s}^{+}(\nu)_{( \pm)}=\Pi^{+} \star \overline{h s}(\nu) \star \Pi^{+} \star \frac{1}{2}(1 \pm \gamma)$ with $\overline{h s}(\nu)$ being the power-series extension of the real form

$$
h s(\nu)=\left\{f \in A q(2 ; \nu) \mid f^{\dagger}=-f\right\},
$$

and $u(\nu ; \sigma, \epsilon)_{( \pm)}=u(\nu \lambda ; \sigma, \epsilon) \star \frac{1}{2}(1 \pm \gamma)$ where

$$
u(\nu ; \sigma, \epsilon)=\left\{f=\sum_{m, n=}^{\infty} f_{n}{ }^{m} P(\nu ; \sigma, \epsilon)_{m}{ }^{n} \mid\left(f_{m}{ }^{n}\right)^{\dagger}=-f_{n}{ }^{n}\right\},
$$


using the simplified notation

$$
P(\nu ; \sigma, \epsilon)_{m}^{n}=\left(P_{\epsilon}^{-,-}\right)_{\lambda_{\epsilon, 2 m+(1+\sigma) / 2}^{\sigma}} \lambda_{\epsilon, 2 n+(1+\sigma) / 2}^{\sigma},
$$

for the generalized projectors defined in (3.43). In this notation, we recall that

$$
\begin{aligned}
P(\nu ; \sigma, \epsilon)_{m}{ }^{n} \star P(\nu ; \sigma, \epsilon)_{m^{\prime}}{ }^{\prime} & =\delta_{m^{\prime}}^{n} P(\nu ; \sigma, \epsilon)_{m}{ }^{n}, \\
\left(P(\nu ; \sigma, \epsilon)_{m}{ }^{n}\right)^{\dagger} & =C \star P(\nu ; \sigma, \epsilon)_{n}{ }^{m} \star C,
\end{aligned}
$$

and for non-critical $\nu$ we have

$$
\mathrm{STr}_{\overline{A q}(2 ; \nu)} P(\nu ; \sigma, \epsilon)_{m}^{n}=-\sigma \delta_{m}^{n} .
$$

As for the fractional spin fields, expanding the $\xi$ dependence as in (2.34) and (2.35), we have

$$
\bar{\Theta}^{\dagger}=-\Theta \star C, \quad \bar{\Sigma}^{\dagger}=-C \star \Sigma .
$$

The equations of motion thus take the form

$$
\begin{array}{llll}
\mathcal{A}_{+} \text {model: } & F^{W}-\Theta \star C \star \Theta^{\dagger}=0, & F^{U}-C \star \Theta^{\dagger} \star \Theta=0, & D \Theta=0, \\
\mathcal{A}_{-} \text {model: } & F^{W}-\Sigma \star C \star \Sigma^{\dagger}=0, & F^{U}-C \star \Sigma^{\dagger} \star \Sigma=0, & D \Sigma=0 .
\end{array}
$$

where $F^{W}=d W+W \star W$ and $F^{U}=d U+U \star U$.

\section{$4.2 \tau$ projections}

One way of truncating further the resulting model is to remove components from $(\psi, \bar{\psi} ; U)$ that have distinct eigenvalues of $w$; for example, one may restrict $U$ to $u(\nu ;+,+)$ and $(\psi, \bar{\psi})$ accordingly, and possibly proceed by further level truncations. However, taking into account the nature of the critical limits, and the fact that truncations by algebra (anti)automorphism would be more natural from the point-of-view of an underlying topological open string, it is more natural to seek to truncate the model by extending the map (2.4) to the fractional spin algebra. This can be achieved by letting $\tau$ act on $\mathrm{Mat}_{2}$ as matrix transposition and taking ${ }^{12}$

$$
\tau(\gamma)=\gamma, \quad \tau(\xi)=i \xi
$$

from which it follows that

$$
\tau\left(\mathbb{X} \star \mathbb{X}^{\prime}\right)=\tau\left(\mathbb{X}^{\prime}\right) \star \tau(\mathbb{X})
$$

Thus, in the $\mathcal{A}_{+}$model we may impose the following $\tau$ projection ${ }^{13}$

$$
\mathcal{A}_{+} \text {model: } \tau(\mathbb{A})=-\mathbb{A},
$$

\footnotetext{
${ }^{12}$ The Clifford algebra $\operatorname{Cliff}_{N}\left(\xi^{i}\right)$ with $N$ fermionic generators $\xi^{i}$ obeying $\left\{\xi^{i}, \xi^{j}\right\}_{\star}=2 \delta^{i j}$ has a graded anti-automorphism defined by $\tau(f \star g)=(-1)^{\epsilon_{s}(f) \epsilon_{s}(g)} \tau(g) \star \tau(f)$ and $\tau\left(\xi^{i}\right)=i \xi^{i}$ where $\epsilon_{s}$ denotes the Grassmann statistics and $\epsilon_{s}\left(\xi^{i}\right)=1$.

${ }^{13}$ The $\mathcal{A}_{-}$model, in which $\tau^{2}(\psi, \bar{\psi})=-(\psi, \bar{\psi})$, cannot be projected using the basic $\tau$ map defined by (2.4), (4.15) and matrix transposition in $\mathrm{Mat}_{2}$.
} 
or, equivalently, in terms of the separate master fields,

$$
\tau(W)=-W, \quad \tau(U)=-U, \quad \tau(\Theta)=-i \bar{\Theta}, \quad \tau(\bar{\Theta})=-i \Theta,
$$

which are indeed consistent with $\tau^{2}=\pi_{q} \pi_{\xi}$ as well as the equations of motion (4.13). The $\tau$ projection removes the fields in $W$ with odd spin and relates the fields in $U$ arising from the gauging of $u(\nu ; \sigma, \epsilon)$ and $u(\nu ; \sigma,-\epsilon)$, respectively, which we denote as

$$
\begin{array}{rlrl}
W & \in \overline{h s}_{0}^{+}(\nu)_{(+)} \oplus \overline{h s}_{0}^{+}(\nu)_{(-)}, & & \overline{h s}_{0}^{+}(\nu)=\left\{f \in \overline{h s}^{+}(\nu) \mid \tau(f)=-f\right\}, \\
U \in \sum_{\sigma}\left[u(\nu ; \sigma)_{(+)} \oplus u(\nu ; \sigma)_{(-)}\right], & u(\nu ; \sigma) & =u(\nu ; \sigma,+)-\tau(u(\nu ; \sigma,+)),
\end{array}
$$

using the fact that $\tau(u(\nu ; \sigma, \epsilon))=u(\nu ; \sigma,-\epsilon)$ and $\tau^{2}(u(\nu ; \sigma, \epsilon))=u(\nu ; \sigma, \epsilon)$.

\subsection{Final form of action and couplings}

Turning to the gauge couplings, up to boundary terms that we will not specify here, the canonically normalized higher spin gravitational and internal parts of the action are defined by

$$
\begin{aligned}
& S_{\mathrm{hs}}[W]=\frac{k_{\mathrm{hs}}}{2 \pi} \int_{M_{3}} \operatorname{Tr}_{h s^{+}(\nu)}\left[\frac{1}{2} W \star d W+\frac{1}{3} W^{\star 3}\right], \\
& S_{\text {int }}[U]=\frac{k_{\text {int }}}{2 \pi} \int_{M_{3}} \operatorname{Tr}_{u(\nu)}\left[\frac{1}{2} U \star d U+\frac{1}{3} U^{\star 3}\right],
\end{aligned}
$$

using trace operations normalized as follows:

$$
\operatorname{Tr}_{h s^{+}(\nu)}\left(J_{a} \star J_{b}\right)=\frac{1}{2} \eta_{a b}, \quad \operatorname{Tr}_{u(\nu)}\left(T_{m}{ }^{n} \star T_{m^{\prime}}{ }^{\prime}\right)=\frac{1}{2} \delta_{m}^{n^{\prime}} \delta_{m^{\prime}}^{n} .
$$

In the gravitational sector, the resulting expressions for Newton's constant and the cosmological constants read

$$
G_{\mathrm{N}}=\frac{\ell_{\mathrm{AdS}}}{4 k_{\mathrm{hs}}}, \quad \Lambda=-\frac{1}{\ell_{\mathrm{AdS}}^{2}},
$$

while the internal level must be integer provided that the base manifold $M_{3}$ is compact and orientable. Comparing (4.23) to

$$
\mathrm{STr}_{A q(2 ; \nu)}\left(J_{a} \star J_{b}\right)=\frac{1}{32}\left(1-\nu^{2}\right)\left(1-\frac{\nu}{3}\right) \eta_{a b},
$$

which follows using (2.21) and

$$
\operatorname{Tr}_{A q(2 ; \nu)}\left(J_{\alpha(2)} \star J_{\beta(2)}\right)=-\frac{1}{4} \epsilon_{\alpha \beta} \epsilon_{\alpha \beta}\left(1-\nu^{2}\right)\left(1-\frac{\nu}{3}\right),
$$

and

$$
\operatorname{STr}_{A q(2 ; \nu)}\left((P(\nu ; \sigma, \epsilon))_{m}{ }^{n} \star(P(\nu ; \sigma, \epsilon))_{m^{\prime}}{ }^{\prime}\right)=-\sigma \delta_{m}^{n^{\prime}} \delta_{m^{\prime}}^{n},
$$

it follows that

$$
\begin{aligned}
\left.\operatorname{STr}_{A q(2 ; \nu)}\right|_{\mathrm{hs}^{+}(\nu)} & =\frac{1}{16}\left(1-\nu^{2}\right)\left(1-\frac{\nu}{3}\right) \operatorname{Tr}_{\mathrm{hs}^{+}(\nu)}, \\
\left.\mathrm{STr}_{A q(2 ; \nu)}\right|_{u(\nu ; \sigma, \epsilon)} & =-2 \sigma \operatorname{Tr}_{u(\nu)} .
\end{aligned}
$$


Hence we obtain

$$
k_{\mathrm{hs}}=\frac{\varkappa}{16}\left(1-\nu^{2}\right)\left(1-\frac{\nu}{3}\right), \quad k_{\mathrm{int}}(u(\nu ; \sigma, \epsilon))=\mp \sigma \varkappa,
$$

where the $\mp$ sign is correlated to the fermion/boson model as one can see from (2.45).

Finally, the $\tau$ projected $\mathcal{A}_{+}$model has the following critical limits:

$$
\begin{array}{r}
\nu=-(2 \ell+1): \mathbb{A} \in\left[\begin{array}{cc}
g l(\ell+1) & (\ell+1, \ell) \\
(\ell, \ell+1) & g l(\ell)
\end{array}\right]=g l(\ell+1 \mid \ell), \\
\nu=2 \ell+1: \mathbb{A} \in\left[\begin{array}{cc}
g l(\ell) & (\ell, \ell+1) \\
(\ell+1, \ell) & g l(\ell+1)
\end{array}\right]=g l(\ell \mid \ell+1) .
\end{array}
$$

Indeed, the gravitational level vanishes, i.e. Newton's constant diverges, in all cases when the $W$ field belongs to either $g l(0)$ or $g l(1)$.

\section{Conclusion}

In summary, we have taken the first steps towards equipping the fractional spin algebra introduced in [8] with a trace operation suitable for the construction of a Chern-Simons model that unifies higher spin gravity, internal gauge fields and fractional spin fields. The model is reminiscent to ordinary Chern-Simons gauged supergraviy [11] and its chirally asymmetric versions [18]. Indeed, as the fractional spin parameter $\nu$ varies, the model interpolates $g l(\ell+1 \mid \ell)$ and $g l(\ell \mid \ell+1)$ models, which arise for the critical values $\nu=-2 \ell-1$ and $\nu=2 \ell+1$, respectively. In particular, we have used the star product formalism to obtain the relation between the gravitational and internal gauge couplings.

In a more abstract sense, the unification of tensorial and fractional spin fields is possible owing to the existence of different realizations of $s l(2, \mathbb{R})$ in the enveloping algebra of the Wigner-deformed Heisenberg algebra (3.2) arising upon choosing different bases. The present model incorporates two such domains of $s l(2, \mathbb{R})$, namely the class of polynomial elements associated with Lorentz tensorial fields, and a class of Gaussian elements associated with fractional spin and internal gauge fields. Along the construction, we have made several choices, whereas a full classification of all possible models would require a more thorough study of the action of $s l(2, \mathbb{R})$ on its enveloping algebra and corresponding supertrace operations. To our best understanding, this remains an open problem, at least in the context of physical model building.

To make further progress, and in particular to enrich the $s l(2, \mathbb{R})$ modules by additional sectors, it would be desirable to use the star product and supertrace operations sensu amplo. To this end, one may use the method employed in this paper, namely to extend the polynomial enveloping algebra by non-polynomial elements corresponding to endomorphisms in lowest-energy spaces spanned by eigenstates of a Hamiltonian belonging to the enveloping algebra of $s l(2, \mathbb{R})$. The salient feature of the resulting extended algebra, namely its associativity and traceability, then follow from the existence of a ground state projector given by a traceable, i.e. real-analytic, element. In the present paper, the latter condition 
amounts to the finiteness of the normalization coefficient in (3.23), which we checked to the first 2 orders in $w$ and which we hope to demonstrate in its entirety in a forthcoming publication within the context of a more convenient realization of the star product.

As for holographic duality, a boundary analysis, possibly along the lines of [23-25], will reveal whether there exist conditions consistent with the standard ones for internal gauge fields and higher spin fields. If so, we expect there to exist a map from boundary states to conformal current algebras, including stress tensors, Kac-Moody currents and intertwining currents $[26,27]$. In particular, it would be interesting to exhibit the boundary states generated by the fractional spin fields, and also to understand whether and how the level of the internal gauge connection, and hence Newton's constant, could be quantized. The holographically dual description may also shed light on the subtle fact that the ideals that arise in critical limits disappear from the action, which amounts to limits of infinite coupling.

The results here presented can be extended to formulations of non-topological theories, namely of the type [28], or by introducing massive anyons (e.g. of $[15,29,30]$ ) in a fractional higher spin gravity background, which we expect to present elsewhere.

\section{Acknowledgments}

We thank Thomas Basile, Xavier Bekaert, Fabien Buisseret, Slava Didenko, Dileep Jatkar, Antal Jevicki and Tomas Prochazka for discussions. For are especially grateful to Thomas Basile for collaboration in proving the relation (3.40). N.B. is supported by the ARC contract No AUWB-2010-10/15-UMONS-1. P.S. is supported by Fondecyt Regular grant No 1140296 and Conicyt grant DPI 20140115. M.V. acknowledges the hospitality of UNAB (Santiago) and CECs (Valdivia).

\section{A Conventions}

We essentially follow the conventions and notation of [12]. We work with metric $\eta_{a b}$ and epsilon symbols $\varepsilon_{a b c}$ and $\varepsilon^{a b c}$ obeying

$$
\eta_{a b}=\operatorname{diag}(-++)_{a b}, \quad \varepsilon^{a b c} \varepsilon_{a^{\prime} b^{\prime} c^{\prime}}=-3 ! \delta_{a^{\prime}}^{[a} \delta_{b^{\prime}}^{b} \delta_{c^{\prime}}^{c]},
$$

and generators

$$
L_{a b}=-\varepsilon_{a b c} J^{c}, \quad J_{a}=\frac{1}{2} \varepsilon_{a b c} L^{b c}
$$

of the Lorentz group obeying

$$
\left[L_{a b}, L^{c d}\right]=4 i \delta_{[b}^{[c} L_{a]}^{d]}, \quad\left[J^{a}, J^{b}\right]=-i \varepsilon^{a b c} J_{c} .
$$

We use real van der Waerden symbols

$$
\left(\tau^{a}\right)_{\alpha \beta}=\left(\tau^{a}\right)_{\beta \alpha}=\left(\left(\tau^{a}\right)_{\alpha \beta}\right)^{\dagger}, \quad \epsilon_{\alpha \beta}=-\epsilon_{\beta \alpha}=\left(\epsilon_{\alpha \beta}\right)^{\dagger}
$$

obeying

$$
\left(\tau^{a}\right)_{\alpha}^{\beta}\left(\tau^{b}\right)_{\beta}^{\gamma}=\eta^{a b} \delta_{\alpha}^{\gamma}+\varepsilon^{a b c}\left(\tau_{c}\right)_{\alpha}^{\gamma}, \quad \epsilon^{\alpha \beta} \epsilon_{\gamma \delta}=2 \delta_{[\gamma}^{\alpha} \delta_{\delta]}^{\beta}
$$


using the convention $q^{\alpha}=\epsilon^{\alpha \beta} q_{\beta}$. A convenient realization in terms of the Pauli matrices is

$$
\begin{aligned}
& \left(\tau^{a}\right)_{\alpha \beta}=\left(-1, \sigma^{1}, \sigma^{3}\right)_{\alpha \beta}, \quad\left(\tau^{a}\right)_{\alpha}{ }^{\beta}=\left(-i \sigma^{2},-\sigma^{3}, \sigma^{1}\right)_{\alpha \beta}, \\
& \epsilon_{\alpha \beta}=\left(-i \sigma^{2}\right)_{\alpha \beta}, \quad \epsilon^{\alpha \beta}=\left(-i \sigma^{2}\right)^{\alpha \beta}, \quad \varepsilon^{012}=1 .
\end{aligned}
$$

In this realization, the operator $w=2 J_{0}=\frac{1}{4}\left(q^{1} \star q^{1}+q^{2} \star q^{2}\right)$, i.e. the Hamiltonian of the deformed harmonic oscillator with deformed momentum $\frac{1}{\sqrt{2}} q^{1}$ and coordinate $\frac{1}{\sqrt{2}} q^{2}$.

Open Access. This article is distributed under the terms of the Creative Commons Attribution License (CC-BY 4.0), which permits any use, distribution and reproduction in any medium, provided the original author(s) and source are credited.

\section{References}

[1] M.A. Vasiliev, Consistent equation for interacting gauge fields of all spins in (3+1)-dimensions, Phys. Lett. B 243 (1990) 378 [INSPIRE].

[2] M.A. Vasiliev, More on equations of motion for interacting massless fields of all spins in (3+1)-dimensions, Phys. Lett. B 285 (1992) 225 [inSPIRE].

[3] V. Bargmann, Irreducible unitary representations of the Lorentz group, Annals Math. 48 (1947) 568.

[4] A.O. Barut and C. Fronsdal, On non-compact groups, II. Representations of the $2+1$ Lorentz group, Proc. Roy. Soc. London A 287 (1965) 532.

[5] J.M. Leinaas and J. Myrheim, On the theory of identical particles, Nuovo Cim. B 37 (1977) 1 [INSPIRE].

[6] F. Wilczek, Quantum Mechanics of Fractional Spin Particles, Phys. Rev. Lett. 49 (1982) 957 [INSPIRE].

[7] S. Forte, Quantum mechanics and field theory with fractional spin and statistics, Rev. Mod. Phys. 64 (1992) 193 [InSPIRE].

[8] N. Boulanger, P. Sundell and M. Valenzuela, Three-dimensional fractional-spin gravity, JHEP 02 (2014) 052 [arXiv: 1312.5700] [INSPIRE].

[9] M.P. Blencowe, A Consistent Interacting Massless Higher Spin Field Theory in D $=(2+1)$, Class. Quant. Grav. 6 (1989) 443 [inSPIRE].

[10] M.A. Vasiliev, Higher Spin Algebras and Quantization on the Sphere and Hyperboloid, Int. J. Mod. Phys. A 6 (1991) 1115 [inSPIRE].

[11] A. Achucarro and P.K. Townsend, A Chern-Simons Action for Three-Dimensional anti-de Sitter Supergravity Theories, Phys. Lett. B 180 (1986) 89 [InSPIRE].

[12] A. Achucarro and P.K. Townsend, Extended Supergravities in $d=(2+1)$ as Chern-Simons Theories, Phys. Lett. B 229 (1989) 383 [INSPIRE].

[13] E.P. Wigner, Do the Equations of Motion Determine the Quantum Mechanical Commutation Relations?, Phys. Rev. 77 (1950) 711.

[14] L.M. Yang, A Note on the Quantum Rule of the Harmonic Oscillator, Phys. Rev. 84 (1951) 788. 
[15] M.S. Plyushchay, Deformed Heisenberg algebra, fractional spin fields and supersymmetry without fermions, Annals Phys. 245 (1996) 339 [hep-th/9601116] [INSPIRE].

[16] M.S. Plyushchay, Deformed Heisenberg algebra with reflection, Nucl. Phys. B 491 (1997) 619 [hep-th/9701091] [INSPIRE].

[17] M. Blagojevic and M. Vasilic, 3 - D gravity with torsion as a Chern-Simons gauge theory, Phys. Rev. D 68 (2003) 104023 [gr-qc/0307078] [INSPIRE].

[18] A. Giacomini, R. Troncoso and S. Willison, Three-dimensional supergravity reloaded, Class. Quant. Grav. 24 (2007) 2845 [hep-th/0610077] [INSPIRE].

[19] C.N. Pope, L.J. Romans and X. Shen, W (infinity) and the Racah-wigner Algebra, Nucl. Phys. B 339 (1990) 191 [inSPIRE].

[20] E.S. Fradkin and V. Ya. Linetsky, Supersymmetric Racah basis, family of infinite dimensional superalgebras, $\mathrm{SU}(\infty+1 \mid \infty)$ and related $2-D$ models, Mod. Phys. Lett. A 6 (1991) 617 [inSPIRE].

[21] A.V. Korybut, Covariant Structure Constants for Deformed Oscillator Algebra, arXiv: 1409.8634 [INSPIRE].

[22] T. Basile and N. Boulanger, unpublished (2015).

[23] M. Henneaux and S.-J. Rey, Nonlinear $W_{\infty}$ as Asymptotic Symmetry of Three-Dimensional Higher Spin Anti-de Sitter Gravity, JHEP 12 (2010) 007 [arXiv: 1008.4579] [INSPIRE].

[24] A. Campoleoni, S. Fredenhagen, S. Pfenninger and S. Theisen, Asymptotic symmetries of three-dimensional gravity coupled to higher-spin fields, JHEP 11 (2010) 007 [arXiv: 1008.4744] [INSPIRE].

[25] M.R. Gaberdiel, R. Gopakumar and A. Saha, Quantum W-symmetry in $A d S_{3}$, JHEP 02 (2011) 004 [arXiv: 1009.6087] [INSPIRE].

[26] D.P. Jatkar and S. Rao, Anyons and Gaussian conformal field theories, Mod. Phys. Lett. A 6 (1991) 289 [InSPIRE].

[27] G.W. Moore and N. Read, Nonabelions in the fractional quantum Hall effect, Nucl. Phys. B 360 (1991) 362.

[28] S.F. Prokushkin and M.A. Vasiliev, Higher spin gauge interactions for massive matter fields in 3 -D AdS space-time, Nucl. Phys. B 545 (1999) 385 [hep-th/9806236] [InSPIRE].

[29] R. Jackiw and V.P. Nair, Relativistic wave equations for anyons, Phys. Rev. D 43 (1991) 1933 [INSPIRE].

[30] J.L. Cortes and M.S. Plyushchay, Linear differential equations for a fractional spin field, J. Math. Phys. 35 (1994) 6049 [hep-th/9405193] [INSPIRE]. 